\title{
Exact Absorbing Boundary Conditions for Periodic Three-Dimensional Structures: Derivation and Implementation in Discontinuous Galerkin Time-Domain Method
}

\author{
Kostyantyn Sirenko ${ }^{\circledR}$, Member, IEEE, Yuriy Sirenko ${ }^{(0)}$, and Hakan Bağc1 ${ }^{\circledR}$, Senior Member, IEEE
}

\begin{abstract}
A discontinuous Galerkin time-domain method (DGTD) enhanced with exact absorbing boundary conditions (EACs) for characterizing transient electromagnetic interactions on periodic three-dimensional (3-D) gratings is proposed. The EACs are derived rigorously and discretized using a high-order scheme in space and time. The periodic boundary conditions (PBCs) under oblique incidence are also discussed. Implementation of the EACs and PBCs within the DGTD framework is described in detail. Numerical results demonstrate that the accuracy of the discretized EACs matches to that of the discretized Maxwell equations. Additionally, the accuracy and efficiency of the DGTD with the EACs are found to be superior to that of the same DGTD with the perfectly matched layers or approximate absorbing boundary conditions.
\end{abstract}

Index Terms-Absorbing boundary conditions, diffraction gratings, discontinuous Galerkin method, periodic structures.

\section{INTRODUCTION}

M ANY of the widely used numerical methods for analyzing electromagnetic wave interactions on gratings are frequency domain techniques [1], [2]. Even though these methods have been proven useful as design tools, the complexity of modern optical, photonic, and electronic devices calls for accurate and efficient three-dimensional (3-D) time-domain solvers. Indeed, 3-D gratings are indispensable components in many

Manuscript received December 16, 2017; revised July 4, 2018; accepted July 17, 2018. Date of publication July 24, 2018; date of current version August 23, 2018. This work was supported in part by the Strategic Research Initiative - Uncertainty Quantification (SRI-UQ) Center, Division of Computer, Electrical, and Mathematical Science and Engineering (CEMSE), King Abdullah University of Science and Technology (KAUST). (Corresponding author: Hakan Bağcl.)

K. Sirenko was with the Division of Computer, Electrical, and Mathematical Science and Engineering (CEMSE), King Abdullah University of Science and Technology (KAUST), Thuwal 23955, Saudi Arabia. He is now with the O. Ya. Usikov Institute for Radiophysics and Electronics, National Academy of Sciences of Ukraine (IRE NASU), Kharkiv 61085, Ukraine (e-mail: k.sirenko@ gmail.com).

Y. Sirenko is with the O. Ya. Usikov Institute for Radiophysics and Electronics, National Academy of Sciences of Ukraine (IRE NASU), Kharkiv 61085, Ukraine, and also with the L. Gumilyov Eurasian National University (ENU), Astana 010000, Kazakhstan (e-mail: yks@ire.kharkov.ua).

H. Bağc1 is with the Division of Computer, Electrical, and Mathematical Science and Engineering (CEMSE), King Abdullah University of Science and Technology (KAUST), Thuwal 23955, Saudi Arabia (e-mail: hakan.bagci@ kaust.edu.sa).

Digital Object Identifier 10.1109/JMMCT.2018.2859315 topical technologies [3]-[6]. Time-domain capabilities are as important as 3-D modeling capabilities. They allow real-time observations of physical processes, can account for nonlinear materials, and provide broadband results in a single execution of the simulation.

The most common time-domain techniques with 3-D modeling capabilities are the finite-difference time-domain (FDTD) [7] and finite-element methods (FEM) [8]. The FDTD is straightforward to implement but typically low-order accurate and works with a uniform/structured spatial discretization. The FEM allows high-order and unstructured discretizations, but it is computationally more expensive in the time domain [9], [10]. Recently, the discontinuous Galerkin time-domain method (DGTD) has become an attractive alternative to the FDTD and FEM for analyzing transient electromagnetic wave interactions [11]-[24]. The numerical flux between discretization elements localizes spatial operations and equips the DGTD with advantages [11] as follows.

1) The mass matrix is block diagonal; its inverse can be obtained and stored very efficiently before time marching.

2) High-order discretizations are easier to implement.

3) Nonconformal discretizations are allowed.

4) $h$-, $p$-, and $h p$-adaptive meshing strategies are easier to implement.

These advantages result in a very efficient solver when combined with an explicit time integration method. Examples of such methods include linear multistepping schemes (AdamsBashfort, Adams-Moulton, Glaser-Rokhlin [25]), and RungeKutta methods [11].

Like any other differential-equation-based technique, the DGTD requires an unbounded physical domain to be truncated into a bounded computation domain. One can use the method of perfectly matched layer (PML) for this purpose. This involves wrapping the computation domain by a layer of lossy material with intrinsic impedance matching that of the computation domain to absorb outgoing waves [21]-[23]. The PML is easy to implement and error controllable up to a certain degree, but it suffers from a couple of drawbacks as follows. It introduces nonnegligible errors, which tend to accumulate as the duration of time marching increases [20], [23]. To match the accuracy of the DGTD, the PML thickness should be increased, which in return increases the computation time and memory requirement [20]. 
The PML can be avoided if one uses exact absorbing boundary conditions (EACs) to truncate unbounded domains [26]-[31]. The EACs are mathematically rigorous and fully error controllable, but their discretization often results in computationally expensive schemes since they require evaluation of "global" spatial and temporal integrals over the computation domain boundaries and the duration of the simulation, respectively. In this paper, the EACs originally formulated in [29]-[31] are used. In contrast to many other nonlocal absorbing boundary conditions, these EACs' efficiency can be improved using well-developed analytical spatial/temporal localization techniques [29]-[31]. One can also use FFT-based acceleration techniques for fast computation of the (nonlocal) temporal convolutions [31]-[34]. These improvements make the EACs an attractive alternative to the PML, especially in simulations that require high accuracy and/or extended time marching [35]-[37]. It should be noted here that the boundaries, where the EACs are enforced, have to coincide with coordinate planes (for example, plane surfaces in Cartesian coordinate system or spherical surfaces in spherical coordinate system). This could make computation domains larger than necessary when the object being analyzed has a certain shape (e.g., a concave object). In this case, one can "hybridize" the DGTD with a time-domain surface integral equation solver [9], [24]. This approach enables the truncation boundary to conform to the surface of the object making the computation domain smaller.

In this paper, the EACs are used together with the periodic boundary conditions (PBCs) to truncate unbounded domains. First, the radiation condition is enforced on outgoing waves. Then, the EACs are derived from the resulting relations by bringing them into specific forms that relate field values to their spatial and temporal derivatives [29]-[31]. The PBCs are derived for obliquely incident fields using the constant wavenumber approach [30], [38], [39]. Being mathematically exact, the EACs do not introduce any errors in theory, but in practice discretization errors are unavoidable. They are discretized using a high-order scheme in space and time, and the numerical flux couples the resulting equations to those obtained by discretizing the Maxwell equations using the DGTD. The PBCs are incorporated into the DGTD via the numerical flux between discretization elements on periodic boundaries. The resulting system of first-order ordinary differential equations is integrated in time using the fourth-order Runge-Kutta method. Additionally, to accelerate the computation of temporal convolutions pertinent to the EACs, a numerically exact blocked FFT scheme is used [31]-[34]. The order of the scheme used for discretizing the EACs matches to that of the DGTD that discretizes the Maxwell equations. Both schemes use high-order polynomial basis functions [20].

Numerical results demonstrate that increasing the order of polynomial expansion increases the overall accuracy of the DGTD with the EACs. Numerical experiments also compare errors introduced by different domain truncation techniques, namely, the EACs, PML, and approximate boundary conditions (ABCs). It is shown that the DGTD with the EACs can obtain five to six digits of accuracy using moderate orders of polynomial expansion. This level of accuracy cannot be achieved by the DGTD with the ABCs or the (thin) PML. Such accuracy is

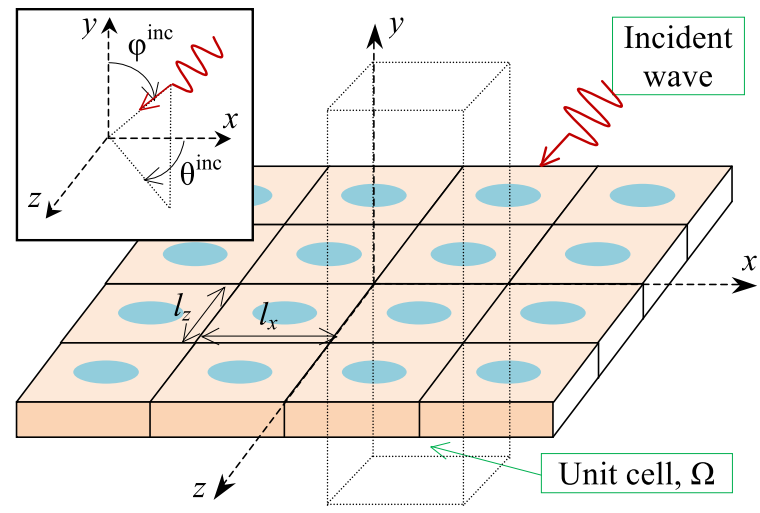

Fig. 1. 3-D periodic grating.

required to avoid error build-up during long time marching required by resonant wave interactions on gratings [2], [35]-[37], [40]. Numerical results also demonstrate the superiority of the DGTD with the EACs over the DGTD with the PML in terms of efficiency for a desired level of accuracy. Additionally, the accuracy of the DGTD with the EACs and (oblique) PCBs is verified against the commercial solver COMSOL, which implements the frequency-domain FEM.

To summarize, the contributions of this paper are threefold.

1) The EACs and (oblique) PBCs are rigorously derived for 3-D gratings.

2) Discretization and incorporation of those within the DGTD framework are discussed in details.

3) Despite its growing popularity for simulations in various branches of physics, the DGTD remains relatively unknown in the optics and photonics communities with only a few papers mentioning it [41]-[43]; and no papers report on the use of the DGTD for analyzing electromagnetic wave interactions on periodic structures.

The paper is organized as follows. Section II describes the mathematical model, derives the EACs and the PBCs. Section III details the DGTD discretization of the Maxwell equations and the discretization and implementation of the EACs within the DGTD framework. Section IV is devoted to numerical experiments.

\section{FORMULATION}

This section details the mathematical model underlying the proposed DGTD solver with the EACs and PBCs. Section II-A introduces the geometry of interest constructed periodically repeating a unit cell. Sections II-B and II-C derive the PBCs under oblique incidence and the EACs, respectively. Section II-D describes the simplified case of normal incidence. Section II-E discusses an efficient way to introduce the excitation using the EACs.

\section{A. Geometry of the Unit Cell}

Consider a 3-D diffraction grating shown in Fig 1. It is constructed by repeating the unit cell $\boldsymbol{\Omega}=\{\mathbf{r}=(x, y, z) \in$ $\left.\mathbb{R}^{3} \mid 0 \leq x<l_{x},-\infty<y<+\infty, 0 \leq z<l_{z}\right\}$ in $x$ and $z$ 


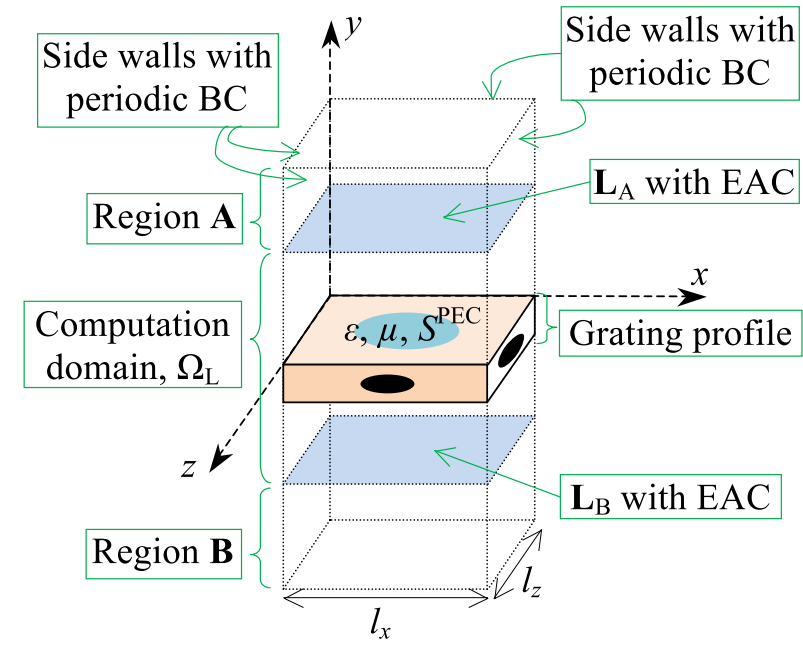

Fig. 2. Unit cell of 3-D periodic grating.

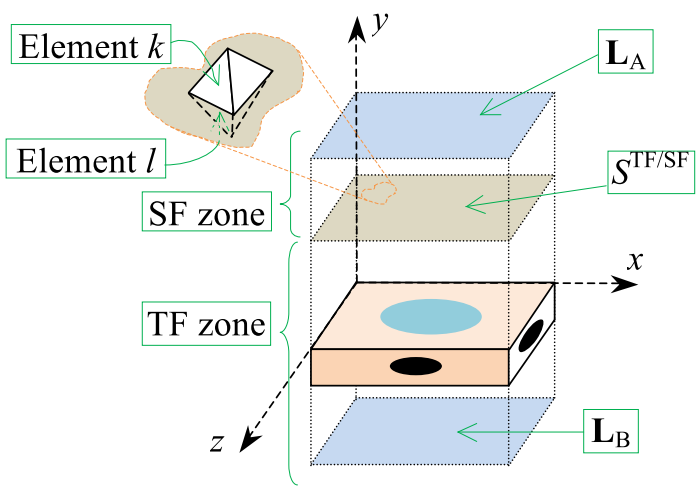

Fig. 3. Computation domain of 3-D grating.

directions with periods $l_{x}$ and $l_{z}$. The grating profile is defined by relative permittivity $\varepsilon(\mathbf{r})$ and relative permeability $\mu(\mathbf{r})$, and possibly by a perfect electrically conducting (PEC) surface $S^{\mathrm{PEC}}$ (see Fig. 2). PEC inclusions $\varepsilon(\mathbf{r})$ and $\mu(\mathbf{r})$ are also periodic in $x$ and $z$ directions with periods $l_{x}$ and $l_{z}$.

To permit numerical modeling using the DGTD, the unbounded physical domain has to be truncated into a bounded computation domain. Truncation in $x$ and $z$ directions from $\mathbb{R}^{3}$ to $\Omega$ is accomplished by imposing the PBCs (see Section II-B) on the side walls of $\Omega$, i.e., at $x=0$ and $x=l_{x}$, and $z=0$ and $z=l_{z}$ (see Fig. 2). Truncation in $y$ direction from $\Omega$ to the computation domain $\Omega_{\mathrm{L}}$ is accomplished by imposing the EACs (see Section II-C) on the virtual boundaries $\mathbf{L}_{\mathrm{A}}$ and $\mathbf{L}_{\mathrm{B}}$ (see Fig. 2). $\boldsymbol{\Omega}_{\mathrm{L}}, \mathbf{L}_{\mathrm{A}}$, and $\mathbf{L}_{\mathrm{B}}$ are defined as $\boldsymbol{\Omega}_{\mathrm{L}}=\left\{\mathbf{r} \in \boldsymbol{\Omega} \mid L_{\mathrm{B}}<y<L_{\mathrm{A}}\right\}, \mathbf{L}_{\mathrm{A}}=\left\{\mathbf{r} \in \boldsymbol{\Omega} \mid y=L_{\mathrm{A}}\right\}$, and $\mathbf{L}_{\mathrm{B}}=\left\{\mathbf{r} \in \boldsymbol{\Omega} \mid y=L_{\mathrm{B}}\right\}$, respectively. The unbounded homogeneous regions external to top and bottom of $\Omega_{\mathrm{L}}$ are defined as $\mathbf{A}=\left\{\mathbf{r} \in \boldsymbol{\Omega} \mid y>L_{\mathrm{A}}\right\}$ and $\mathbf{B}=\left\{\mathbf{r} \in \boldsymbol{\Omega} \mid y<L_{\mathrm{B}}\right\}$. With these definitions, $\boldsymbol{\Omega}=\boldsymbol{\Omega}_{\mathrm{L}} \cup \mathbf{A} \cup \mathbf{B} \cup \mathbf{L}_{\mathrm{A}} \cup \mathbf{L}_{\mathrm{B}}$. Additionally, $\Omega_{\mathrm{L}}$ is divided into the total field (TF) and the scattered field (SF) regions by surface $S^{\mathrm{TF} / \mathrm{SF}}$ (see Fig. 3). It is assumed that the grating profile is entirely embedded in the TF region and $S^{\mathrm{TF} / \mathrm{SF}}$ is placed between $\mathbf{L}_{\mathrm{A}}$ and the profile.

\section{B. PBCs Under Oblique Incidence}

The derivation of the PBCs enforced on the side walls of $\Omega_{\mathrm{L}}$ is trivial when the electromagnetic field possesses the same periodicity as the unit cell and material properties. But this happens only when the excitation is normally incident on the unit cell (see Section II-D). In the general case, a grating is excited by an obliquely incident field; its direction of arrival is defined by the angles $\varphi^{\text {inc }}$ and $\theta^{\text {inc }}$ (see Fig. 1). Under obliquely incident fields, the PBCs require "future" values of the fields, and therefore cannot be directly used in numerical simulations [38], [44]. If the incident field extends to infinity in space (as in excitation with a plane-wave) and is obliquely incident, then this causality problem cannot be alleviated. But if the incident field is bounded in space, the issue of causality can be solved using various approaches. The most widely used is the transformed-variables approach [44], [45]: the field components are imparted with the periodicity via the transformation of variables, but this also changes the governing equations. This change in the equations makes the derivation of the EACs impossible. As an alternative, in this paper, the constant wavenumber approach is used [30], [38], [39]: the fields are represented in terms of time-dependent quasi-periodic functions. This approach does not require any variable transformation and, thus, does not avert the derivation of the EACs as described next.

Let $\mathbf{U}_{\text {phys }}^{\text {inc }}(\mathbf{r}, t) \in\left\{\mathbf{E}_{\text {phys }}^{\text {inc }}(\mathbf{r}, t), \mathbf{H}_{\text {phys }}^{\text {inc }}(\mathbf{r}, t)\right\}$ represent the physical incident electromagnetic field. It is assumed that $\mathbf{U}_{\text {phys }}^{\text {inc }}(\mathbf{r}, t)$ is bounded in space, arrives onto the grating from $\mathbf{A}$, and $\mathbf{U}_{\text {phys }}^{\text {inc }}\left(\mathbf{r}_{L_{\mathrm{A}}}, 0\right)=0, \mathbf{r}_{L_{\mathrm{A}}}=\left(x, L_{\mathrm{A}}, z\right)$. $\mathbf{U}_{\text {phys }}^{\text {inc }}(\mathbf{r}, t)$ is expanded as

$$
\begin{aligned}
& \mathbf{U}_{\text {phys }}^{\text {inc }}(\mathbf{r}, t)=\int_{-\infty}^{\infty} \int_{-\infty}^{\infty} \mathbf{U}^{\text {inc }}\left(\mathbf{r}, t, \Phi_{x}, \Phi_{z}\right) d \Phi_{x} d \Phi_{z} \\
& =\int_{-\infty}^{\infty} \int_{-\infty}^{\infty} \tilde{\mathbf{U}}^{\text {inc }}\left(y, t, \Phi_{x}, \Phi_{z}\right) e^{2 \pi i \Phi_{x} x / l_{x}} e^{2 \pi i \Phi_{z} z / l_{z}} d \Phi_{x} d \Phi_{z} .
\end{aligned}
$$

Here, $\mathbf{U}^{\text {inc }}\left(\mathbf{r}, t, \Phi_{x}, \Phi_{z}\right)$ is quasi-periodic in $x$ and $z$ directions with periods $l_{x}$ and $l_{z}$

$$
\begin{aligned}
& \mathbf{U}^{\mathrm{inc}}\left(x+l_{x}, y, z, t, \Phi_{x}, \Phi_{z}\right)=e^{2 \pi i \Phi_{x}} \mathbf{U}^{\mathrm{inc}}\left(x, y, z, t, \Phi_{x}, \Phi_{z}\right) \\
& \mathbf{U}^{\mathrm{inc}}\left(x, y, z+l_{z}, t, \Phi_{x}, \Phi_{z}\right)=e^{2 \pi i \Phi_{z}} \mathbf{U}^{\mathrm{inc}}\left(x, y, z, t, \Phi_{x}, \Phi_{z}\right)
\end{aligned}
$$

$\tilde{\mathbf{U}}^{\text {inc }}\left(y, t, \Phi_{x}, \Phi_{z}\right)$ represents $y$-dependent component of $\mathbf{U}^{\text {inc }}$ $\left(\mathbf{r}, t, \Phi_{x}, \Phi_{z}\right), \mathbf{U}^{\text {inc }}\left(\mathbf{r}, t, \Phi_{x}, \Phi_{x}\right) \in\left\{\mathbf{E}^{\text {inc }}\left(\mathbf{r}, t, \Phi_{x}, \Phi_{x}\right), \mathbf{H}^{\text {inc }}\right.$ $\left.\left(\mathbf{r}, t, \Phi_{x}, \Phi_{x}\right)\right\}$, and $\mathbf{E}^{\text {inc }}\left(\mathbf{r}, t, \Phi_{x}, \Phi_{x}\right)$ and $\mathbf{H}^{\text {inc }}\left(\mathbf{r}, t, \Phi_{x}, \Phi_{x}\right)$ are incident fields that satisfy the Maxwell equations. Therefore, they can be used to excite $\Omega$. The fields generated under this excitation also satisfy the Maxwell equations and possess the same periodicity as follows:

$$
\begin{aligned}
\nabla \times \mathbf{H}(\mathbf{r}, t) & =\varepsilon(\mathbf{r}) \partial_{t} \mathbf{E}(\mathbf{r}, t) \\
\nabla \times \mathbf{E}(\mathbf{r}, t) & =-\mu(\mathbf{r}) \partial_{t} \mathbf{H}(\mathbf{r}, t) \\
\left.\mathbf{U}(\mathbf{r}, t)\right|_{t=0} & =0,\left.\quad \partial_{t} \mathbf{U}(\mathbf{r}, t)\right|_{t=0}=0, \mathbf{r}=(x, y, z) \in \mathbf{\Omega} \\
\mathbf{U}\left(l_{x}, y, z, t\right) & =e^{2 \pi i \Phi_{x}} \mathbf{U}(0, y, z, t), 0 \leq z<l_{z} \\
\mathbf{U}\left(x, y, l_{z}, t\right) & =e^{2 \pi i \Phi_{z}} \mathbf{U}(x, y, 0, t), 0 \leq x<l_{x} .
\end{aligned}
$$


Here, $\mathbf{U}(\mathbf{r}, t) \in\{\mathbf{E}(\mathbf{r}, t), \mathbf{H}(\mathbf{r}, t)\}$ and represents the total field in the TF region and the scattered field in the SF region (see Fig. 3). The explicit dependence on $\Phi_{x}$ and $\Phi_{z}$ is dropped from here on, as problem (1) is solved for each given value of $\Phi_{x}$ and $\Phi_{z}$ separately. Zero initial conditions are used since it is assumed that $\mathbf{U}^{\text {inc }}(\mathbf{r}, t)$ arrives onto the grating from $\mathbf{A}$ and $\mathbf{U}^{\text {inc }}\left(\mathbf{r}_{L}, 0\right)=0$. It is assumed that the boundary conditions on $S^{\mathrm{PEC}}$ are satisfied. It should be noted here that in (1), unit-free normalized Maxwell equations are used [11]. Once $\mathbf{E}(\mathbf{r}, t)$ and $\mathbf{H}(\mathbf{r}, t)$ are obtained by solving (1) for a set of $\Phi_{x}$ and $\Phi_{z}$, the solution of physical problem, $\mathbf{U}_{\text {phys }}(\mathbf{r}, t) \in$ $\left\{\mathbf{E}_{\text {phys }}(\mathbf{r}, t), \mathbf{H}_{\text {phys }}(\mathbf{r}, t)\right\}$ [which is generated due to the physical incident field $\left.\mathbf{U}_{\text {phys }}^{\text {inc }}(\mathbf{r}, t)\right]$ is reconstructed from $\mathbf{E}(\mathbf{r}, t)$ and $\mathbf{H}(\mathbf{r}, t)$ using

$$
\mathbf{U}_{\text {phys }}(\mathbf{r}, t)=\int_{-\infty}^{\infty} \int_{-\infty}^{\infty} \mathbf{U}\left(\mathbf{r}, t, \Phi_{x}, \Phi_{z}\right) d \Phi_{x} d \Phi_{z} .
$$

It should be noted here that for each value of $\Phi_{x}$ and $\Phi_{z}$, each frequency component of the excitation has its own direction of arrival, $\varphi$ and $\theta$, given by $\Phi_{x}=k l_{x} \sin \varphi \cos \theta /(2 \pi)$ and $\Phi_{z}=k l_{z} \sin \varphi \sin \theta /(2 \pi)$, where $k$ is the wavenumber.

\section{EACs}

In this section, the EACs enforced on $\mathbf{L}_{\mathrm{A}}$ and $\mathbf{L}_{\mathrm{B}}$ (see Figs. 2 and 3) are derived. The derivation of the EAC on $\mathbf{L}_{\mathrm{A}}$ is same as that of the $\mathrm{EAC}$ on $\mathbf{L}_{\mathrm{B}}$; therefore, only the derivation of the EAC on $\mathbf{L}_{\mathrm{A}}$ is detailed here.

In $\mathbf{A} \cup \mathbf{L}_{\mathrm{A}}$, which is assumed to be entirely embedded in the SF region, every component of the field $U(\mathbf{r}, t)$ satisfies the homogenous wave equation with zero initial conditions as follows:

$$
\begin{aligned}
\partial_{t}^{2} U(\mathbf{r}, t) & =\partial_{x}^{2} U(\mathbf{r}, t)+\partial_{y}^{2} U(\mathbf{r}, t)+\partial_{z}^{2} U(\mathbf{r}, t) \\
U(\mathbf{r}, 0) & =\left.\partial_{t} U(\mathbf{r}, t)\right|_{t=0}=0, \mathbf{r}=(x, y, z) \in \mathbf{A} \cup \mathbf{L}_{\mathrm{A}} \\
U\left(l_{x}, y, z, t\right) & =e^{2 \pi i \Phi_{x}} U(0, y, z, t), 0 \leq z<l_{z} \\
U\left(x, y, l_{z}, t\right) & =e^{2 \pi i \Phi_{z}} U(x, y, 0, t), 0 \leq x<l_{x} .
\end{aligned}
$$

To solve (2), $U(\mathbf{r}, t)$ is expanded in terms of spatial harmonics as follows:

$$
U(\mathbf{r}, t)=\sum_{n=-\infty}^{\infty} \sum_{m=-\infty}^{\infty} u_{n m}(y, t) f_{n m}(x, z)
$$

Here, $u_{n m}(y, t)$ are the unknown amplitudes and $f_{n m}(x, z)$ are orthonormal transverse spatial harmonics (eigenfunctions). Using the orthogonality of spatial harmonics, $u_{n m}(y, t)$ can be related to $U(\mathbf{r}, t)$ as follows:

$$
u_{n m}(y, t)=\int_{0}^{l_{x}} \int_{0}^{l_{z}} U(\mathbf{r}, t) f_{n m}^{*}(x, z) d x d z .
$$

Here, “*” stands for the complex conjugate. Inserting (3) into (2) yields, for each $f_{n m}(x, z)$, a set of homogenous equations that are easily solved as

$$
f_{n m}(x, z)=e^{i \alpha_{n} x} e^{i \beta_{m} z} / \sqrt{l_{x} l_{z}}
$$

where $\alpha_{n}=2 \pi\left(n+\Phi_{x}\right) / l_{x}$ and $\beta_{m}=2 \pi\left(m+\Phi_{z}\right) / l_{z}$ with $\lambda_{n m}^{2}=\alpha_{n}^{2}+\beta_{m}^{2}$ being the eigenvalues corresponding to $f_{n m}(x, z)$. Inserting (3) into (2) also yields an initial value problem satisfied by $u_{n m}(y, t)$ as follows:

$$
\begin{gathered}
-\partial_{t}^{2} u_{n m}(y, t)+\partial_{y}^{2} u_{n m}(y, t)-\lambda_{n m}^{2} u_{n m}(y, t)=0 \\
u_{n m}(y, 0)=\left.\partial_{t} u_{n m}(y, t)\right|_{t=0}=0, y \geq L_{\mathrm{A}} .
\end{gathered}
$$

Solution of (6) is carried out in the spectral domain [29]-[31]: taking the cosine Fourier transform of (6) yields a Cauchy problem. This Cauchy problem is solved analytically using the concept of generalized functions and fundamental solution. Finally, taking the inverse Fourier transform of the solution of the Cauchy problem yields

$$
\begin{aligned}
u_{n m}(y, t)= & -\int_{0}^{t-y+L_{\mathrm{A}}} J_{0}\left(\lambda_{n m} \sqrt{(t-\tau)^{2}-\left(y-L_{\mathrm{A}}\right)^{2}}\right) \\
& \times\left.\partial_{y} u_{n m}(y, \tau)\right|_{y=L_{\mathrm{A}}} d \tau, y \geq L_{\mathrm{A}} .
\end{aligned}
$$

Here, $J_{0}($.$) is the zeroth-order Bessel function. Setting y=L_{\mathrm{A}}$ in (7) yields

$$
u_{n m}\left(L_{\mathrm{A}}, t\right)=-\left.\int_{0}^{t} J_{0}\left(\lambda_{n m}[t-\tau]\right) \partial_{y} u_{n m}(y, \tau)\right|_{y=L_{\mathrm{A}}} d \tau
$$

To obtain a relation between $\partial_{t} u_{n m}\left(L_{A}, t\right)$ and $\left.\partial_{y} u_{n m}(y, t)\right|_{y=L_{A}}$, which is compatible with the DGTD implementation, (8) is mathematically manipulated. Taking the Laplace transform of (8) and rearranging the terms in the resulting expression yield

$$
\begin{aligned}
& s \tilde{u}_{n m}\left(L_{\mathrm{A}}, s\right)+\left.\partial_{y} \tilde{u}_{n m}(y, s)\right|_{y=L_{\mathrm{A}}} \\
& \quad=-\left(\sqrt{\left(s^{2}+\lambda_{n m}^{2}\right)}-s\right) \tilde{u}_{n m}\left(L_{\mathrm{A}}, s\right) .
\end{aligned}
$$

Here, $\tilde{u}_{n m}(y, s)$ is the Laplace transform image of $u_{n m}(y, t)$. Taking the inverse Laplace transform of (9) yields the desired relation between $\partial_{t} u_{n m}\left(L_{A}, t\right)$ and $\left.\partial_{y} u_{n m}(y, t)\right|_{y=L_{A}}$ as follows:

$$
\begin{aligned}
\partial_{t} & u_{n m}\left(L_{\mathrm{A}}, t\right)+\left.\partial_{y} u_{n m}(y, t)\right|_{y=L_{\mathrm{A}}} \\
& =-\lambda_{n m} \int_{0}^{t} \frac{J_{1}\left(\lambda_{n m}[t-\tau]\right)}{t-\tau} u_{n m}\left(L_{\mathrm{A}}, \tau\right) d \tau .
\end{aligned}
$$

Here, $J_{1}($.$) is the first-order Bessel function. Using (3) and (4),$ a relation between $\partial_{t} U\left(\mathbf{r}_{L_{\mathrm{A}}}, t\right)$ and $\left.\partial_{y} U(\mathbf{r}, t)\right|_{\mathbf{r}=\mathbf{r}_{L_{A}}}$ on $\mathbf{L}_{\mathrm{A}}$ is obtained from (10) as

$$
\begin{aligned}
\partial_{t} U\left(\mathbf{r}_{L_{\mathrm{A}}}, t\right)+\left.\partial_{y} U(\mathbf{r}, t)\right|_{\mathbf{r}=\mathbf{r}_{L_{\mathrm{A}}}} \\
=-\sum_{n=-\infty}^{\infty} \sum_{m=-\infty}^{\infty} \lambda_{n m} f_{n m}(x, z) \int_{0}^{t} \frac{J_{1}\left(\lambda_{n m}[t-\tau]\right)}{t-\tau} \\
\quad \times \int_{0}^{l_{x}} \int_{0}^{l_{z}} U\left(\tilde{\mathbf{r}}_{L_{\mathrm{A}}}, \tau\right) f_{n m}^{*}(\tilde{x}, \tilde{z}) d \tilde{x} d \tilde{z} d \tau \\
\mathbf{r}_{L_{\mathrm{A}}}=\left(x, L_{\mathrm{A}}, z\right), 0 \leq x<l_{x}, 0 \leq z<l_{z} \\
\tilde{\mathbf{r}}_{L_{\mathrm{A}}}=\left(\tilde{x}, L_{\mathrm{A}}, \tilde{z}\right) .
\end{aligned}
$$


Following the same steps, a similar relation can be derived between $\partial_{t} U\left(\mathbf{r}_{L_{\mathrm{B}}}, t\right)$ and $\left.\partial_{y} U(\mathbf{r}, t)\right|_{\mathbf{r}=\mathbf{r}_{L_{\mathrm{B}}}}$ on $\mathbf{L}_{\mathrm{B}}$ as

$$
\begin{aligned}
& \partial_{t} U\left(\mathbf{r}_{L_{\mathrm{B}}}, t\right)-\left.\partial_{y} U(\mathbf{r}, t)\right|_{\mathbf{r}=\mathbf{r}_{L_{\mathrm{B}}}} \\
&=-\sum_{n=-\infty}^{\infty} \sum_{m=-\infty}^{\infty} \lambda_{n m} f_{n m}(x, z) \int_{0}^{t} \frac{J_{1}\left(\lambda_{n m}[t-\tau]\right)}{t-\tau} \\
& \quad \times \int_{0}^{l_{x}} \int_{0}^{l_{z}} U\left(\tilde{\mathbf{r}}_{L_{\mathrm{B}}}, \tau\right) f_{n m}^{*}(\tilde{x}, \tilde{z}) d \tilde{x} d \tilde{z} d \tau \\
& \quad \mathbf{r}_{L_{\mathrm{A}}}=\left(x, L_{\mathrm{B}}, z\right), 0 \leq x<l_{x}, 0 \leq z<l_{z} \\
& \tilde{\mathbf{r}}_{L_{\mathrm{B}}}=\left(\tilde{x}, L_{\mathrm{B}}, \tilde{z}\right) .
\end{aligned}
$$

Equations (11) and (12) establish a relation between the boundary values of the field and its spatial and temporal derivatives. Thus, they can be enforced on $\mathbf{L}_{\mathrm{A}}$ and $\mathbf{L}_{\mathrm{B}}$ as the EACs: fields that arrive onto $\mathbf{L}_{\mathrm{A}}$ and $\mathbf{L}_{\mathrm{B}}$ from $\Omega_{\mathrm{L}}$ are neither deformed nor reflected back into $\Omega_{\mathrm{L}}$ and behave as if they are absorbed by $\mathbf{L}_{\mathrm{A}}$ and $\mathbf{L}_{\mathrm{B}}$. EACs (11) and (12) are derived rigorously and they are free from any approximations. This makes their errorcontrollable discretization possible as described in Section III.

It should be noted here that the solution of problem (1) provides the field distribution inside $\Omega_{\mathrm{L}}$ in the close proximity of grating profile. To obtain the far-field response, one must compute the fields scattered in $\mathbf{A}$ and $\mathbf{B}$. This can be achieved by finding a transport operator that relates the fields on $\mathbf{L}_{\mathrm{A}}$ and $\mathbf{L}_{\mathrm{B}}$ to the fields in $\mathbf{A}$ and $\mathbf{B}$, respectively. The fields in $\mathbf{A}$ can be expressed in terms of the fields on $\mathbf{L}_{\mathrm{A}}$ by using (3) and (4) in (7) [30], [31]. The fields in $\mathbf{B}$ can be expressed in terms of the fields on $\mathbf{L}_{\mathrm{B}}$ in a similar way.

\section{Normal Incidence}

Under a normally incident field $\left(\varphi^{\text {inc }}=\theta^{\text {inc }}=0\right)$, problem (1) simplifies to

$$
\begin{aligned}
\nabla \times \mathbf{H}(\mathbf{r}, t) & =\varepsilon(\mathbf{r}) \partial_{t} \mathbf{E}(\mathbf{r}, t) \\
\nabla \times \mathbf{E}(\mathbf{r}, t) & =-\mu(\mathbf{r}) \partial_{t} \mathbf{H}(\mathbf{r}, t) \\
\left.\mathbf{U}(\mathbf{r}, t)\right|_{t=0} & =0,\left.\partial_{t} \mathbf{U}(\mathbf{r}, t)\right|_{t=0}=0, \mathbf{r}=(x, y, z) \in \mathbf{\Omega} \\
\mathbf{U}\left(l_{x}, y, z, t\right) & =\mathbf{U}(0, y, z, t), 0 \leq z<l_{z} \\
\mathbf{U}\left(x, y, l_{z}, t\right) & =\mathbf{U}(x, y, 0, t), 0 \leq x<l_{x} .
\end{aligned}
$$

Here, $\mathbf{U}(\mathbf{r}, t) \in\{\mathbf{E}(\mathbf{r}, t), \mathbf{H}(\mathbf{r}, t)\}$ directly represents the physical fields since $\Phi_{x}=\Phi_{z}=0$. The EACs (11) and (12) also hold with physical fields, but the transverse spatial harmonics $f_{n m}(x, z)$ should be replaced with

$$
f_{n m}(x, z)=g\left(\alpha_{n} x\right) g\left(\beta_{m} z\right) / \sqrt{l_{x} l_{z}}
$$

where $g(a x)=\cos (a x)$ for $a \geq 0, g(a x)=\sin (a x)$ for $a<$ $0, \alpha_{n}=2 \pi n / l_{x}$, and $\beta_{m}=2 \pi m / l_{z}$. It should also be noted here that the conjugation in (11) and (12) can be dropped since $f_{n m}(x, z)$ are now real.

\section{E. Excitation Through the Virtual Boundary $\mathbf{L}_{\mathrm{A}}$}

If the excitation is introduced via the TF/SF formulation [20][22] (see Section III-A), part of $\Omega_{\mathrm{L}}$ between $\mathbf{L}_{\mathrm{A}}$ and $S^{\mathrm{TF} / \mathrm{SF}}$ becomes "unused" space (see Fig. 3). Computation domain $\Omega_{\mathrm{L}}$ can be shrunk further if $S^{\mathrm{TF} / \mathrm{SF}}$ can be made to coincide with $\mathbf{L}_{\mathrm{A}}$, and the excitation is introduced using the EAC on $\mathbf{L}_{\mathrm{A}}$ [30], [31] ( $\boldsymbol{\Omega}_{\mathrm{L}}$ coincides with the TF region). In this case, the EAC (11) should be updated as

$$
\begin{aligned}
\partial_{t} U\left(\mathbf{r}_{L_{\mathrm{A}}}, t\right)-2 \partial_{t} U^{\mathrm{inc}}\left(\mathbf{r}_{L_{\mathrm{A}}}, t\right)=-\left.\partial_{y} U(\mathbf{r}, t)\right|_{\mathbf{r}=\mathbf{r}_{L_{\mathrm{A}}}} \\
\quad-\sum_{n=-\infty}^{\infty} \sum_{m=-\infty}^{\infty} \lambda_{n m} f_{n m}(x, z) \int_{0}^{t} \frac{J_{1}\left([t-\tau] \lambda_{n m}\right)}{t-\tau} \\
\quad \times \int_{0}^{l_{x}} \int_{0}^{l_{z}}\left[U\left(\tilde{\mathbf{r}}_{L_{\mathrm{A}}}, \tau\right)-2 U^{\mathrm{inc}}\left(\tilde{\mathbf{r}}_{L_{\mathrm{A}}}, \tau\right)\right] \\
\quad \times f_{n m}^{*}(\tilde{x}, \tilde{z}) d \tilde{x} d \tilde{z} d \tau \\
\mathbf{r}_{L_{\mathrm{A}}}=\left(x, L_{\mathrm{A}}, z\right), 0 \leq x<l_{x}, 0 \leq z<l_{z}, \tilde{\mathbf{r}}_{L_{\mathrm{A}}}=\left(\tilde{x}, L_{\mathrm{A}}, \tilde{z}\right) .
\end{aligned}
$$

Here, $U^{\text {inc }}(\mathbf{r}, t)$ and $U(\mathbf{r}, t)$ represent any one of the components of the incident and total fields, respectively.

\section{DISCRETIZATION}

This section describes the discretization of the Maxwell equations supported with the time-domain PBCs and EACs as derived in Section II.

\section{A. Discretization of the Maxwell Equations}

To solve problem (1) numerically, a nodal DGTD scheme is used. The DGTD discretizes $\Omega_{\mathrm{L}}$ into $N_{e}$ nonoverlapping tetrahedral elements. On element $k$, each component of $\mathbf{E}(\mathbf{r}, t)$ and $\mathbf{H}(\mathbf{r}, t)$ is expanded as

$$
\begin{aligned}
E_{u}(\mathbf{r}, t) \cong \sum_{i=1}^{N_{p}} E_{u}^{k}\left(\mathbf{r}_{i}^{k}, t\right) \ell_{i}(\mathbf{r}) & =\sum_{i=1}^{N_{p}} E_{u, i}^{k}(t) \ell_{i}(\mathbf{r}) \\
H_{u}(\mathbf{r}, t) \cong \sum_{i=1}^{N_{p}} H_{u}^{k}\left(\mathbf{r}_{i}^{k}, t\right) \ell_{i}(\mathbf{r}) & =\sum_{i=1}^{N_{p}} H_{u, i}^{k}(t) \ell_{i}(\mathbf{r})
\end{aligned}
$$

where $u \in\{x, y, z\}, k=1, \ldots, N_{e}, \ell_{i}(\mathbf{r})$ is the multidimensional Lagrange interpolation polynomials of order $p, N_{p}=$ $(p+1)(p+2)(p+3) / 6$ is the number of nodes in each element, and $\mathbf{r}_{i}^{k}$ denotes the location of Gauss-Lobatto nodes within element $k$ [11]. Here, $E_{u, i}^{k}(t)$ and $H_{u, i}^{k}(t)$ are the unknowns to be solved for. Inserting (14) into the Maxwell equations in (1), testing the resulting equation with $\ell_{i}(\mathbf{r})$, and applying integration by parts twice yields a strong form that can be solved numerically using the DGTD [11] as follows:

$$
\begin{aligned}
\varepsilon^{k} \partial_{t} \bar{E}^{k}(t) & =\bar{D}^{k} \times \bar{H}^{k}(t)+\bar{N}^{k} \bar{F}_{E}^{k}(t) \\
-\mu^{k} \partial_{t} \bar{H}^{k}(t) & =\bar{D}^{k} \times \bar{E}^{k}(t)+\bar{N}^{k} \bar{F}_{H}^{k}(t)
\end{aligned}
$$

where superscript $k$ represents quantities associated with element $k$. Here, $\mu^{k}$ and $\varepsilon^{k}$ are relative permeability and permittivity, which are assumed constant over element $k, \bar{E}^{k}(t)=$ $\left[\bar{E}_{x}^{k}(t), \bar{E}_{y}^{k}(t), \bar{E}_{z}^{k}(t)\right]^{T}$ and $\bar{H}^{k}(t)=\left[\bar{H}_{x}^{k}(t), \bar{H}_{y}^{k}(t), \bar{H}_{z}^{k}(t)\right]^{T}$ are $3 N_{p} \times 1$ vectors consisting of three $N_{p} \times 1$ vectors of the unknowns $\left[\bar{E}_{u}^{k}(t)\right]_{i}=E_{u, i}^{k}(t)$ and $\left[\bar{H}_{u}^{k}(t)\right]_{i}=H_{u, i}^{k}(t)$, 


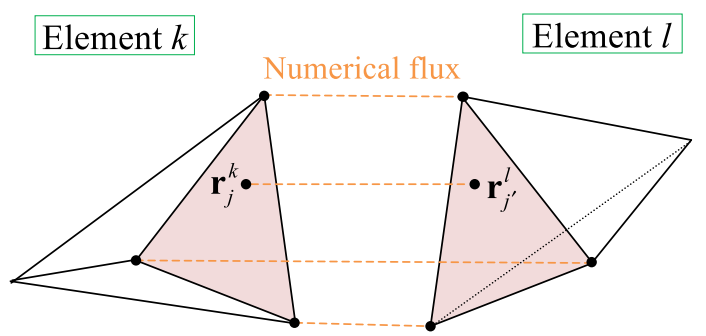

Fig. 4. Numerical flux "connects" neighboring elements.

$\bar{D}^{k}=\left[\bar{D}_{x}^{k}, \bar{D}_{y}^{k}, \bar{D}_{z}^{k}\right]$ is a $3 N_{p} \times N_{p}$ matrix consisting of three $N_{p} \times N_{p}$ differentiation matrices $\bar{D}_{u}^{k}$, and $\bar{N}^{k}=\operatorname{diag}$ $\left\{\left[\left(\bar{M}^{k}\right)^{-1} \bar{L}^{k},\left(\bar{M}^{k}\right)^{-1} \bar{L}^{k},\left(\bar{M}^{k}\right)^{-1} \bar{L}^{k}\right]\right\}$ is a $3 N_{p} \times 3 N_{p}$ blockdiagonal matrix, where each block is a matrix product of the inverse of $N_{p} \times N_{p}$ mass matrix $\bar{M}^{k}$ and $N_{p} \times N_{p}$ lift matrix $\bar{L}^{k}$. Nonzero entries of $\bar{D}_{u}^{k}, \bar{M}^{k}$, and $\bar{L}^{k}$ are [11]

$$
\begin{aligned}
{\left[\bar{D}_{u}^{k}\right]_{i j} } & =\partial_{u} \ell_{j}\left(\mathbf{r}_{i}^{k}\right), i=1, \ldots, N_{p}, j=1, \ldots, N_{p} \\
{\left[\bar{M}^{k}\right]_{i j} } & =\int_{\boldsymbol{\Omega}^{k}} \ell_{i}(\mathbf{r}) \ell_{j}(\mathbf{r}) d \mathbf{r}, i=1, \ldots, N_{p}, j=1, \ldots, N_{p} \\
{\left[\bar{L}^{k}\right]_{i j} } & =\int_{\partial \boldsymbol{\Omega}^{k}} \ell_{i}(\mathbf{r}) \ell_{j}(\mathbf{r}) d \mathbf{r}, i=1, \ldots, N_{p}
\end{aligned}
$$

$j \in\left\{j \mid \mathbf{r}_{j}^{k} \in \partial \boldsymbol{\Omega}^{k}, j=1, \ldots, N_{p}\right\}$

where $\boldsymbol{\Omega}^{k}$ is the volumetric support of element $k$, and $\partial \boldsymbol{\Omega}^{k}$ is the surface of $\boldsymbol{\Omega}^{k}$. In (15), $\bar{F}_{H}^{k}(t)=\left[\bar{F}_{H, x}^{k}(t), \bar{F}_{H, y}^{k}(t), \bar{F}_{H, z}^{k}(t)\right]^{T}$ and $\bar{F}_{E}^{k}(t)=\left[\bar{F}_{E, x}^{k}(t), \bar{F}_{E, y}^{k}(t), \bar{F}_{E, z}^{k}(t)\right]^{T}$ are $3 N_{p} \times 1$ vectors, each of which consists of three $N_{p} \times 1$ vectors $\bar{F}_{E, u}^{k}(t)$ and $\bar{F}_{H, u}^{k}(t)$, known as the upwind numerical flux between element $k$ and its neighbor. Entries of $\bar{F}_{E, u}^{k}(t)$ and $\bar{F}_{H, u}^{k}(t)$ are [11]

$$
\begin{aligned}
{\left[\bar{F}_{E, u}^{k}(t)\right]_{j} } & =\left[\mathbf{n}_{j} \times\left(Z^{l} \Delta \mathbf{H}_{j}^{k}-\mathbf{n}_{j} \times \Delta \mathbf{E}_{j}^{k}\right)\right]_{u} /\left(Z^{k}+Z^{l}\right) \\
{\left[\bar{F}_{H, u}^{k}(t)\right]_{j} } & =\left[\mathbf{n}_{j} \times\left(Y^{l} \Delta \mathbf{E}_{j}^{k}-\mathbf{n}_{j} \times \Delta \mathbf{H}_{j}^{k}\right)\right]_{u} /\left(Y^{k}+Y^{l}\right) .
\end{aligned}
$$

Here, $j \in\left\{j \mid \mathbf{r}_{j}^{k} \in \partial \boldsymbol{\Omega}^{k} \cap \partial \boldsymbol{\Omega}^{l}, j=1, \ldots, N_{p}\right\}, l$ is the index of any neighbor of element $k$ (see Fig. 4), $Z^{v}=1 / Y^{v}=$ $\sqrt{\left(\mu^{v} / \varepsilon^{v}\right)}, v \in\{k, l\}, \mathbf{n}_{j}$ is the outward unit normal of $\partial \boldsymbol{\Omega}^{k}$ at $\mathbf{r}_{j}^{k},\left(\Delta \mathbf{E}_{j}^{k}\right)_{u}=E_{u, j^{\prime}}^{l}(t)-E_{u, j}^{k}(t)$, and $\left(\Delta \mathbf{H}_{j}^{k}\right)_{u}=$ $H_{u, j^{\prime}}^{l}(t)-H_{u, j}^{k}(t)$ for $j^{\prime} \in\left\{j^{\prime} \mid \mathbf{r}_{j^{\prime}}^{l}=\mathbf{r}_{j}^{k}, j=1, \ldots, N_{p}, j^{\prime}=\right.$ $\left.1, \ldots, N_{p}\right\}$, where $j^{\prime}$ runs through the indices of element $l$ 's nodes and $\mathbf{r}_{j}^{k}$ and $\mathbf{r}_{j^{\prime}}^{l}$ point to the same physical location that belongs to elements $k$ and $l$ (see Fig. 4). The cross products in (15) are defined as

$$
\begin{aligned}
& \bar{D}^{k} \times \bar{E}^{k}(t)= {\left[\begin{array}{l}
\bar{D}_{y}^{k} \bar{E}_{z}^{k}(t)-\bar{D}_{z}^{k} \bar{E}_{y}^{k}(t) \\
\bar{D}_{z}^{k} \bar{E}_{x}^{k}(t)-\bar{D}_{x}^{k} \bar{E}_{z}^{k}(t) \\
\bar{D}_{x}^{k} \bar{E}_{y}^{k}(t)-\bar{D}_{y}^{k} \bar{E}_{x}^{k}(t)
\end{array}\right] } \\
& \bar{D}^{k} \times \bar{H}^{k}(t)=\left[\begin{array}{c}
\bar{D}_{y}^{k} \bar{H}_{z}^{k}(t)-\bar{D}_{z}^{k} \bar{H}_{y}^{k}(t) \\
\bar{D}_{z}^{k} \bar{H}_{x}^{k}(t)-\bar{D}_{x}^{k} \bar{H}_{z}^{k}(t) \\
\bar{D}_{x}^{k} \bar{H}_{y}^{k}(t)-\bar{D}_{y}^{k} \bar{H}_{x}^{k}(t)
\end{array}\right] .
\end{aligned}
$$

Boundary conditions on $S^{\mathrm{PEC}}$ are enforced by modifying the numerical flux defined on the faces of elements that are in contact with $S^{\mathrm{PEC}}$. This is achieved by setting in (16), $Z^{l}=$ $Z^{k}, Y^{l}=Y^{k},\left(\Delta \mathbf{E}_{j}^{k}\right)_{u}=-2 E_{u, j}^{k}(t)$, and $\left(\Delta \mathbf{H}_{j}^{k}\right)_{u}=0, j \in$ $\left\{j \mid \mathbf{r}_{j}^{k} \in \partial \boldsymbol{\Omega}^{k} \cap S^{\mathrm{PEC}}\right\}[11]$.

The numerical flux can be used to introduce an excitation via the TF/SF formulation [20]-[22]. It is assumed that profile is fully enclosed in the TF region (see Fig. 3). Let $\mathbf{E}^{\text {inc }}(\mathbf{r}, t)=\left[E_{x}^{\text {inc }}(\mathbf{r}, t), E_{y}^{\text {inc }}(\mathbf{r}, t), E_{z}^{\text {inc }}(\mathbf{r}, t)\right]$ and $\mathbf{H}^{\text {inc }}(\mathbf{r}, t)=\left[H_{x}^{\text {inc }}(\mathbf{r}, t), H_{y}^{\text {inc }}(\mathbf{r}, t), H_{z}^{\text {inc }}(\mathbf{r}, t)\right]$ represent the electric and magnetic field of the incident wave. The difference in fields on $S^{\mathrm{TF} / \mathrm{SF}}$ is accounted by modifying the numerical flux on the faces of elements in contact with $S^{\mathrm{TF} / \mathrm{SF}}$. This is achieved by setting in (16) $\left(\Delta \mathbf{E}_{j}^{k}\right)_{u}=E_{u, j^{\prime}}^{l}(t)-E_{u, j}^{k}(t) \pm$ $E_{u}^{\text {inc }}\left(\mathbf{r}_{j^{\prime}}^{l}, t\right),\left(\Delta \mathbf{H}_{j}^{k}\right)_{u}=H_{u, j^{\prime}}^{l}(t)-H_{u, j}^{k}(t) \pm H_{u}^{\text {inc }}\left(\mathbf{r}_{j^{\prime}}^{l}, t\right), j \in$ $\left\{j \mid \mathbf{r}_{j}^{k} \in \partial \boldsymbol{\Omega}^{k} \cap \partial \boldsymbol{\Omega}^{l} \cap S^{\mathrm{TF} / \mathrm{SF}}\right\}, \quad j^{\prime} \in\left\{j^{\prime} \mid \mathbf{r}_{j^{\prime}}^{l}=\mathbf{r}_{j}^{k}\right\}$. Here, the sign " + " is selected if element $k$ is in the TF region, and "_" if it is in the SF region. In this formulation, if element $k$ is in the TF region, then element $l$ is in the SF region, and vice versa (see Fig. 3).

The PBCs on the side walls of $\Omega_{\mathrm{L}}$ (see Fig. 2) are enforced by modifying the numerical flux on the faces of elements in contact with these side walls. Let $S^{x=0}, S^{x=l_{x}}, S^{z=0}$, and $S^{z=l_{z}}$ represent the side walls of $\Omega_{\mathrm{L}}$ lying in the planes $x=0, x=l_{x}, z=0$, and $z=l_{z}$. Assuming that elements $k$ and $l$ are in contact with the opposite walls, (16) is modified as $\left(\Delta \mathbf{E}_{j}^{k}\right)_{u}=\mathrm{e}^{ \pm 2 \pi i \Phi_{v}} E_{u, j^{\prime}}^{l}(t)-E_{u, j}^{k}(t),\left(\Delta \mathbf{H}_{j}^{k}\right)_{u}=\mathrm{e}^{ \pm 2 \pi i \Phi_{v}}$ $H_{u, j^{\prime}}^{l}(t)-H_{u, j}^{k}(t), j \in\left\{j \mid \mathbf{r}_{j}^{k} \in \partial \boldsymbol{\Omega}^{k} \cap S^{v=0}\right.$ or $\mathbf{r}_{j}^{k} \in \partial \boldsymbol{\Omega}^{k} \cap$ $\left.S^{v=l_{v}}\right\}, j^{\prime} \in\left\{j^{\prime} \mid\left(\mathbf{r}_{j^{\prime}}^{l}\right)_{y}=\left(\mathbf{r}_{j}^{k}\right)_{y}\right.$ and $\left.\left(\mathbf{r}_{j^{\prime}}^{l}\right)_{v^{\prime}}=\left(\mathbf{r}_{j}^{k}\right)_{v^{\prime}}\right\}, v, v^{\prime} \in$ $\{x, z\}, v \neq v^{\prime}$. The sign " + " is selected if element $k$ is in contact with $S^{x=l_{x}}$ or $S^{z=l_{z}}$, and "_" if it is in contact with $S^{x=0}$ or $S^{z=0}$. It should be noted here that elements $k$ and $l$ are not in physical contact, but still the numerical flux connects them.

\section{B. Discretization of the EACs}

To obtain the maximum efficiency and accuracy of the DGTD with the EACs, the order of the discretization of the EACs should match that of the DGTD. The EACs (11), (12), and (13) are discretized in the same way; therefore, only the discretization of the EAC (11) on $\mathbf{L}_{\mathrm{A}}$ is described step by step here next.

1) Let $k$ represent the index of any element that have a face on $\mathbf{L}_{\mathrm{A}}$. It is assumed that $\mathbf{L}_{\mathrm{A}}$ is discretized by a surface mesh generated from the faces of these elements. Let $l$ represent the index of the face that touches element $k$ (see Fig. 5). Each face has $N_{s}=(p+1)(p+2) / 2$ nodes on it, and each element has $N_{p}=(p+1)(p+2)(p+3) / 6$ nodes in it, $p$ is the polynomial order of the DGTD. The locations of nodes on face $l$ are represented with $\mathbf{r}_{j^{\prime}}^{l}, j^{\prime}=$ $1, \ldots, N_{s}$, and the locations of nodes within element $k$ are represented with $\mathbf{r}_{j}^{k}, j=1, \ldots, N_{p}$ (see Fig. 5).

2) Let $\hat{\bar{U}}^{l}(t) \in\left\{\hat{\bar{E}}_{u}^{l}(t), \hat{\bar{H}}_{u}^{l}(t)\right\}$ represent an $N_{s} \times 1$ vector that stores exact boundary field samples accounted for by the EAC (11) at $\mathbf{r}_{j^{\prime}}^{l}, j^{\prime}=1, \ldots, N_{s}$. Additionally, let $\bar{U}^{k}(t) \in\left\{\bar{E}_{u}^{k}(t), \bar{H}_{u}^{k}(t)\right\}$ represent an $N_{p} \times 1$ vector that stores samples of fields accounted for by the Maxwell 


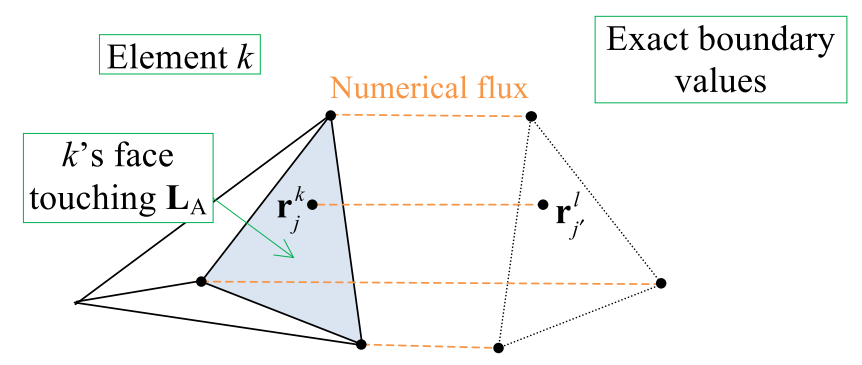

Fig. 5. Numerical flux "enforces" EAC.

equations in (1) at $\mathbf{r}_{j}^{k}, j=1, \ldots, N_{p}$. The boundary field samples $\hat{\bar{U}}^{l}(t)$ should not be confused with the samples accounted for by the Maxwell equations at the nodes of element $k$ that are located on its face that coincides with the face $l$, i.e., $\left[\bar{U}^{k}(t)\right]_{j}, j \in\left\{j \mid \mathbf{r}_{j}^{k} \in \partial \boldsymbol{\Omega}^{k} \cap \mathbf{L}_{\mathrm{A}}\right\}$. These are two different sets even though the nodes refer to the same physical locations.

3) To discretize the spatial derivative in (11), $\partial_{y} U$ $\left.(\mathbf{r}, t)\right|_{\mathbf{r}=\mathbf{r}_{L_{A}}}$, DGTD's differentiation matrix $\bar{D}_{y}^{k}$ is used. $\bar{D}_{y}^{k}$ is an $N_{p} \times N_{p}$ matrix that computes the samples of fields' $y$-derivative at all nodes of element $k$, i.e., at $\mathbf{r}_{j}^{k}$, $j=1, \ldots, N_{p}$, using the field samples at the same nodes. Let

$$
\left[\tilde{\bar{U}}^{k}(t)\right]_{j}= \begin{cases}{\left[\hat{\bar{U}}^{l}(t)\right]_{j^{\prime}}} & \text { for } \mathbf{r}_{j}^{k}=\mathbf{r}_{j^{\prime}}^{l} \\ {\left[\bar{U}^{k}(t)\right]_{j}} & \text { otherwise }\end{cases}
$$

Then, the multiplication $\bar{D}_{y}^{k} \tilde{\bar{U}}^{k}(t)$ produces an $N_{p} \times 1$ vector that stores samples of $\tilde{U}^{k}(t)$ 's $y$-derivatives at $\mathbf{r}_{j}^{k}$, $j=1, \ldots, N_{p}$. Let

$$
\left[\bar{Q}^{k}\right]_{j^{\prime} j}=\left\{\begin{array}{cc}
1 & \text { for } \mathbf{r}_{j}^{k}=\mathbf{r}_{j^{\prime}}^{l} \\
0 & \text { otherwise }
\end{array}\right.
$$

represent an $N_{s} \times N_{p}$ sparse matrix that selects the field samples at $\mathbf{r}_{j^{\prime}}^{l}, j^{\prime}=1, \ldots, N_{s}$. Then, the samples of $\left.\partial_{y} U(\mathbf{r}, t)\right|_{\mathbf{r}=\mathbf{r}_{L_{\mathrm{A}}}}$ at $\mathbf{r}_{j^{\prime}}^{l}, j^{\prime}=1, \ldots, N_{s}$ are approximated by $\bar{Q}^{k} \bar{D}_{y}^{k} \tilde{\bar{U}}^{k}(t)$.

4) The spatial integral in (11) over $\tilde{x}$ and $\tilde{z}$ is evaluated numerically. To achieve this on each face discretizing $\mathbf{L}_{\mathrm{A}}$, 2-D quadrature rules are used [46]. It should be noted here that the quadrature points and the DGTD nodes on these faces do not coincide [47]. Therefore, an interpolation scheme is used to compute the field samples at quadrature points from the samples at the face nodes. Let $\bar{I}^{l}$ represent an $N_{q} \times N_{s}$ interpolation matrix associated with integration on face $l$ as follows:

$$
\bar{I}^{l}=\bar{V}^{l}\left(\bar{W}^{l}\right)^{-1}
$$

where $\bar{V}^{l}$ and $\bar{W}^{l}$ are $N_{q} \times N_{s}$ and $N_{s} \times N_{s}$ Vandermonde matrices with entries $\left[\bar{V}^{l}\right]_{i i^{\prime}}=\ell_{i^{\prime}}\left(\mathbf{q}_{i}^{l}\right)$ and $\left[\bar{W}^{l}\right]_{j^{\prime} i^{\prime}}=\ell_{i^{\prime}}\left(\mathbf{r}_{j^{\prime}}^{l}\right)$, respectively. Index $i^{\prime}$ selects only those DGTD polynomials $\ell(\mathbf{r})$ that are nonzero on face $l$ (there are $N_{s}$ such polynomials [11]) and $\mathbf{q}_{i}^{l}, i=1, \ldots, N_{q}$ represent the quadrature points. Using the interpolation matrix, the spatial integration in (11) is approximated as

$$
\int_{0}^{l_{x}} \int_{0}^{l_{z}} U\left(\tilde{\mathbf{r}}_{L_{\mathrm{A}}}, \tau\right) f_{n m}^{*}(\tilde{x}, \tilde{z}) d \tilde{x} d \tilde{z} \cong \sum_{l} \bar{w}^{l} \bar{I}^{l} \bar{f}_{n m}^{l *} \hat{\bar{U}}^{l}(\tau) .
$$

Here, index $l$ runs over the indices of all faces that discretize $\mathbf{L}_{\mathrm{A}}, \bar{w}^{l}$ is an $N_{q} \times N_{q}$ diagonal matrix that stores the quadrature weights associated with $\mathbf{q}_{i}^{l}$, and $\bar{f}_{n m}^{l *}$ is an $N_{q} \times 1$ vector that stores the samples of conjugated spatial harmonics at $\mathbf{q}_{i}^{l}:\left[\bar{f}_{n m}^{l * *}\right]_{i}=f_{n m}^{*}\left(\mathbf{q}_{i}^{l}\right)$. It should be noted here that $N_{q}$ determines the order of integration rule, which is selected to match the order of the DGTD polynomials.

5) The summations over $m$ and $n$ in (11) are truncated to finite numbers of terms, $2 N_{m}+1$ and $2 N_{n}+1$, respectively. $N_{m}$ and $N_{n}$ are chosen in such a way that the highest spectral content of outgoing waves passing through $\mathbf{L}_{\mathrm{A}}$ are captured by the highest (fastest varying) harmonic included in the summations. Spectral content of the outgoing waves depends both on the properties of the excitation and geometry. The evanescent harmonics should also be included if the virtual boundaries are placed close to the profile. The harmonic with index $n$ and $m$ is identified as propagating if $k^{2} \geq \lambda_{n m}^{2}$, and as evanescent if $k^{2}<\lambda_{n m}^{2}$. Here, $\lambda_{n m}$ is the eigenvalue defined in (5) and $k$ is the wavenumber. It should be emphasized here that setting $N_{m}$ and $N_{n}$ arbitrarily large may introduce unexpected errors in the solution since the discretization will not be able to accurately resolve the spatial variations of higher harmonics.

These five steps result in spatially discretized version of the EAC (11) as follows:

$$
\begin{aligned}
\partial_{t} \hat{\bar{U}}^{l}(t)= & -\bar{Q}^{k} \bar{D}_{y}^{k} \tilde{\bar{U}}^{k}(t) \\
& -\sum_{n=-N_{n}}^{N_{n}} \sum_{m=-N_{m}}^{N_{m}} \lambda_{n m} \bar{f}_{n m}^{l} \int_{0}^{t} \frac{J_{1}\left(\lambda_{n m}[t-\tau]\right)}{t-\tau} \\
& \times \sum_{l^{\prime}} \bar{w}^{l^{\prime}} \bar{I}^{l^{\prime}} \bar{f}_{n m}^{l^{\prime} *} \hat{\bar{U}}^{l^{\prime}}(\tau) d \tau
\end{aligned}
$$

where indices $l$ and $l^{\prime}$ run through the indices of all faces on $\mathbf{L}_{\mathrm{A}}$, and $k$ is the index of the DGTD element that touches face $l$. Similarly, the spatially discretized version of the EAC (12) is

$$
\begin{aligned}
\partial_{t} \hat{\bar{U}}^{l}(t)= & \bar{Q}^{k} \bar{D}_{y}^{k} \tilde{\bar{U}}^{k}(t) \\
& -\sum_{n=-N_{n}}^{N_{n}} \sum_{m=-N_{m}}^{N_{m}} \lambda_{n m} \bar{f}_{n m}^{l} \int_{0}^{t} \frac{J_{1}\left(\lambda_{n m}[t-\tau]\right)}{t-\tau} \\
& \times \sum_{l^{\prime}} \bar{w}^{l^{\prime}} \bar{I}^{l^{\prime}} \bar{f}_{n m}^{l^{\prime} *} \hat{\bar{U}}^{l^{\prime}}(\tau) d \tau
\end{aligned}
$$

where indices $l$ and $l^{\prime}$ run through the indices of all faces on $\mathbf{L}_{\mathrm{B}}$. The spatially discretized version of the EAC (13) is obtained in 
the same way as

$$
\begin{aligned}
\partial_{t} \hat{\bar{U}}^{l}(t) & -2 \partial_{t} \bar{U}_{\text {inc }}^{l}(t)=-\bar{Q}^{k} \bar{D}_{y}^{k} \tilde{\bar{U}}^{k}(t) \\
& -\sum_{n=-N_{n}}^{N_{n}} \sum_{m=-N_{m}}^{N_{m}} \lambda_{n m} \bar{f}_{n m}^{l} \int_{0}^{t} \frac{J_{1}\left(\lambda_{n m}[t-\tau]\right)}{t-\tau} \\
& \times \sum_{l^{\prime}} \bar{w}^{l^{\prime}} \bar{I}^{l^{\prime}} \bar{f}_{n m}^{l^{\prime} *}\left[\hat{\bar{U}}^{l^{\prime}}(\tau)-2 \bar{U}_{\text {inc }}^{l^{\prime}}(\tau)\right] d \tau .
\end{aligned}
$$

Here, $\bar{U}_{\text {inc }}^{l}(t)$ is an $N_{s} \times 1$ vector that stores the samples of the incident fields at $\mathbf{r}_{j^{\prime}}^{l}, j^{\prime}=1, \ldots, N_{s}$.

The spatially discretized EACs (17)-(19) are coupled to the spatially discretized Maxwell equations (15) using the numerical flux. This is achieved by updating $\Delta \mathbf{E}_{j}^{k}$ and $\Delta \mathbf{H}_{j}^{k}$ in (16) with the exact boundary field component samples provided by (17)-(19). The values of $\Delta \mathbf{E}_{j}^{k}$ and $\Delta \mathbf{H}_{j}^{k}$, where index $k$ runs through the indices of all elements that have a face on the virtual boundaries, are set to $\left(\Delta \mathbf{E}_{j}^{k}\right)_{u}=\hat{E}_{u, j^{\prime}}^{l}(t)-E_{u, j}^{k}(t)$ and $\left(\Delta \mathbf{H}_{j}^{k}\right)_{u}=\hat{H}_{u, j^{\prime}}^{l}(t)-H_{u, j}^{k}(t)$ for $j, j^{\prime} \in\left\{j, j^{\prime} \mid \mathbf{r}_{j^{\prime}}^{l}=\mathbf{r}_{j}^{k}, j=\right.$ $\left.1, \ldots, N_{p}, j^{\prime}=1, \ldots, N_{p}\right\}, l$ is the index of face that touches element $k$ (see Fig. 5). Here, $\hat{E}_{u, j^{\prime}}^{l}(t)$ and $\hat{H}_{u, j^{\prime}}^{l}(t)$ are the samples of the boundary field components stored in $\hat{\bar{U}}^{l}(t)$ and provided by (17)-(19), and $E_{u, j}^{k}(t)$ and $H_{u, j}^{k}(t)$ are the samples of the DGTD boundary field components stored in $\bar{E}^{k}(t)$ and $\bar{H}^{k}(t)$ and provided by the Maxwell equations (15).

The semidiscrete system of equations obtained by coupling the discretized Maxwell equations (15) and (17)-(19) forms a system of ordinary differential equations in time. This system of equations is integrated to obtain the samples of $\bar{E}^{k}(t), \bar{H}^{k}(t)$, and $\hat{\bar{U}}^{l}(t)$. In this paper, the fourth-order explicit Runge-Kutta method is used [11]. The integral over $\tau$ in EACs (17)-(19) can be computed using any numerical integration technique that has the same order as the time integration of the ordinary differential equation system. In this paper, the Simpson rule is used since its order matches to that of the Runge-Kutta method.

It should be noted here that the EACs used in this paper are nonlocal in space and time in their current form. However, their efficiency could be improved by using analytical spatial/temporal localization techniques without sacrificing from accuracy [29]-[31]. In this paper, since the number of (surface) discretization elements on $\mathbf{L}_{\mathrm{A}}$ and $\mathbf{L}_{\mathrm{B}}$ is significantly smaller than the number of (volume) discretization elements in $\Omega_{\mathrm{L}}$, the spatial localization is not utilized. However, the computation of the temporal convolutions in (17)-(19) is accelerated using a blocked-FFT scheme without introducing any additional errors [31]-[34]. This reduces the cost of computing these convolutions to $O\left(N_{t} \log ^{2} N_{t}\right)$ from $O\left(N_{t}^{2}\right)$, where $N_{t}$ is the number of time steps [31]-[34]. The application of this blocked-FFT scheme does not change how the EACs (17)-(19) are coupled to the Maxwell equations (15).

\section{NumericAl RESUlts}

This section details numerical experiments that demonstrate the accuracy and efficiency of the proposed DGTD with the EACs and PBCs. Section IV-A characterizes the dependence of the DGTD solution error on various parameters including the

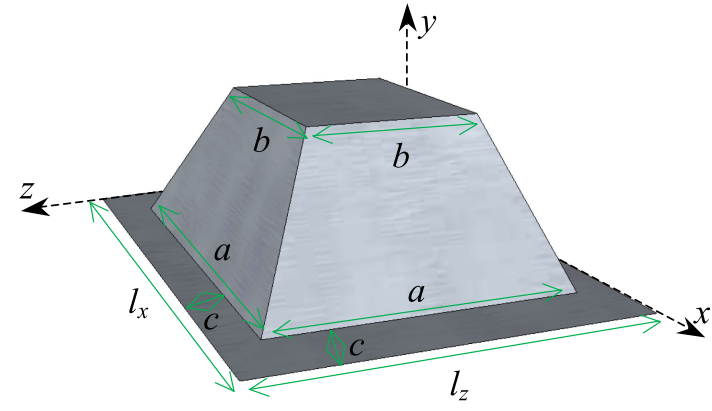

Fig. 6. Unit cell profile of the grating studied in Section IV-A.

method of truncation (EAC, PML, or ABC), discretization order, distance between absorbing boundaries and grating profile, and length of simulation duration. It compares the accuracy and efficiency of introducing the excitation through the EACs to those of the classical TF/SF approach. Additionally, in Section IV-A, the accuracy of the DGTD with the EACs and (oblique) PCBs is verified against the commercial solver COMSOL. Section IV-B demonstrates the applicability of the proposed solver to geometrically complicated structures. In all simulations, the excitation is a quasi-periodic electromagnetic field, as described in Section II-B. The components of the electric field of this excitation are given by $E_{x}^{\text {inc }}(r, t)=E_{y}^{\text {inc }}(r, t)=0$ and $E_{z}^{\text {inc }}(r, t)=$ $U_{z}^{\text {inc }}\left(r, t, \Phi_{x}, \Phi_{z}\right)=E(t) e^{2 \pi i \Phi_{x} x / l_{x}} e^{2 \pi i \Phi_{z} z / l_{z}}$, where $E(t)$ is the time signature of the excitation.

All simulations are carried out on an eight-core workstation with $2.67 \mathrm{GHz}$ CPUs and $23.4 \mathrm{~GB}$ of RAM. The DGTD is implemented in $\mathrm{C}$ and parallelized using MPI.

\section{A. Accuracy}

The unit cell profile of the geometry analyzed in this section is shown in Fig. 6. The profile is a truncated PEC pyramid centered on a plane PEC substrate. The lengths of pyramid's edges at bottom and top bases are $a=0.42$ and $b=0.28$, the height of pyramid is $h=0.5$, and the distance between pyramid's edge at bottom base and side wall is $c=0.08$. The periods are $l_{x}=$ $l_{z}=0.5$

Numerical simulations are carried out using the DGTD with the following four different domain truncation techniques: EAC (11); EAC (13); ABC; and PML. These solvers are named "DGTD-EAC," "DGTD-EACe," "DGTD-ABC," and "DGTDPML" in the rest of this section. The reference results are obtained using the same DGTD with a large computation domain truncated with PEC boundaries. This solver is named "DGTDref."

The DGTD-EAC and DGTD-EACe truncate the summations in the EACs (11) and (13) with five terms, i.e., $N_{n}=N_{m}=2$, resulting in a total of 25 harmonics. Frequencies corresponding to the cutoff wavenumbers of the harmonics with $n=m= \pm 2$ are always beyond the maximum frequency of $E(t)$ used in the simulations; thus, increasing $N_{n}$ and $N_{m}$ further does not increase the accuracy of the EAC truncation.

The DGTD-ABC implements the first-order Engquist-Majda $\mathrm{ABC}$, i.e., it replaces the $\operatorname{EAC}$ (13) with $\partial_{t} U\left(\mathbf{r}_{L_{\mathrm{A}}}, t\right)=$ $-\left.\partial_{y} U(\mathbf{r}, t)\right|_{\mathbf{r}=\mathbf{r}_{L_{\mathrm{A}}}}[20]$. 


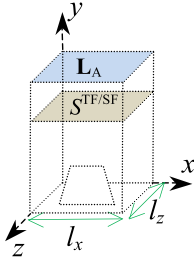

(a)

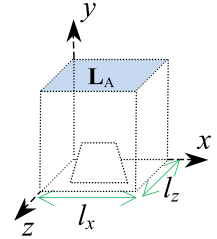

(b)

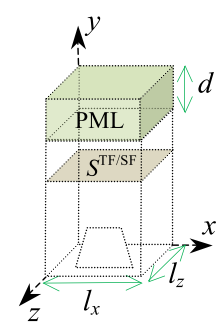

(c)

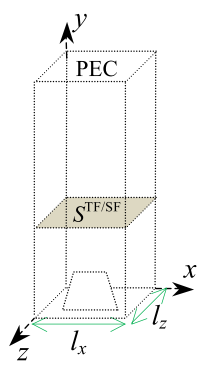

(d)
Fig. 7. Computation domains. (a) For the DGTD-EAC and DGTD-ABC (b) For the DGTD-EACe, (c) For the DGTD-PML, and (d) For the DGTD-ref.

The DGTD-PML implements the uniaxial PML [22], [23], where PML loss parameter increases linearly within the layer from 0 to 20. Two different layer thicknesses are used: $d=0.25$ and $d=0.5$. Outer boundary of the layer is PEC.

For the DGTD-EAC, DGTD-ABC, DGTD-PML, and DGTD-ref, the excitation is implemented using the TF/SF method (see Section III-B) by introducing $S^{\mathrm{TF} / \mathrm{SF}}$ between $\mathbf{L}_{\mathrm{A}}$ and the profile. For the DGTD-EACe, the excitation is introduced on $\mathbf{L}_{\mathrm{A}}$ (see Section II-E). All solvers use the same PBCs (see Section II-B) and discretization of the computation domain.

As a measure of accuracy, the global $L^{2}$-error is used as follows:

$$
\operatorname{err}(t)=\sqrt{\frac{1}{N_{\mathrm{err}}} \int_{\boldsymbol{\Omega}_{\text {err }}}\left|E_{z}(\mathbf{r}, t)-E_{z}^{\mathrm{ref}}(\mathbf{r}, t)\right|^{2} d \mathbf{r} .}
$$

Here, $E_{z}(\mathbf{r}, t)$ is the solution computed by the DGTD-EAC, DGTD-EACe, DGTD-ABC, or DGTD-PML, and $E_{z}^{\mathrm{ref}}(\mathbf{r}, t)$ is the reference solution computed by the DGTD-ref in a computation domain with dimensions $[0 ; 0.5] \times[0 ; 6] \times[0 ; 0.5]$, which is truncated with the PEC boundary at $y=6$. This domain is large enough to prevent reflections coming back into the domain of error computation $\Omega_{\mathrm{err}}$ within the duration of the simulation. $N_{\text {err }}$ is the number of discretization elements in $\Omega_{\text {err }}$. This measure of accuracy accounts for the error of domain truncation and does not take into account the error of the DGTD itself. Computation domains for the DGTD-EAC, DGTD-ABC, DGTDEACe, DGTD-PML, and DGTD-ref are shown in Fig. 7(a)-(d), respectively.

First, the dependence of the error on the polynomial order $p$ is characterized. For this set of simulations, $E(t)=$ $2 e^{-(t-1.5)^{2} / 0.13} \cos (15[t-1.5])$ is a modulated Gaussian pulse. Two sets of values $\Phi_{x}=\Phi_{z}=0$ (normal incidence) and $\Phi_{x}=\Phi_{z}=0.1$ (oblique incidence) are considered. The duration of the simulations is 7.5. The computation domains for the DGTD-EAC, DGTD-ABC, and DGTD-PML are $[0 ; 0.5] \times$ $[0 ; 2] \times[0 ; 0.5],[0 ; 0.5] \times[0 ; 2] \times[0 ; 0.5]$, and $[0 ; 0.5] \times[0 ; 2+$ $d] \times[0 ; 0.5] . S^{\mathrm{TF} / \mathrm{SF}}$ is located at $y=1.5$. The numbers of the elements in the computation domains are 3357, 3357, 3847 $(d=0.25)$, and $4357(d=0.5)$. Fig. 8 plots err $(7.5)$ computed on $\Omega_{\mathrm{err}}=[0 ; 0.5] \times[0 ; 2] \times[0 ; 0.5]$ against $p$. It is clearly seen that the error of the EAC truncation decreases with growing $p$, while the errors of $\mathrm{ABC}$ and PML truncations remain virtually the same for any $p$. This means that the accuracy of the EAC truncation naturally matches the overall accuracy of the DGTD.

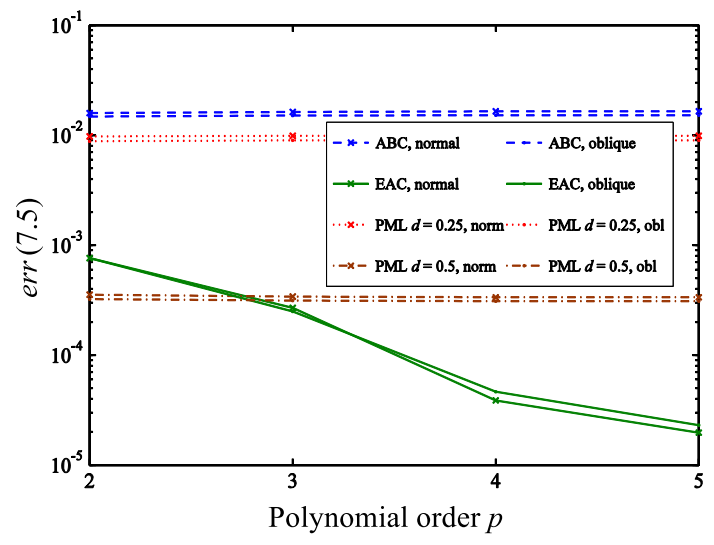

Fig. 8. err(7.5) computed by DGTD-EAC, DGTD-ABC, and DGTD-PML versus $p$ for normal and oblique incidence.

For larger values of $p$, the overall accuracy of the simulations with the ABC or PML truncation is limited by the accuracy of the truncation and increasing $p$ does not result in more accurate simulations. For smaller values of $p$, the PML accuracy becomes excessively high since the overall accuracy is limited by the error of the DGTD. Fig. 8 also shows that the errors are on the same level for normal and oblique excitations.

Next, it is shown that the DGTD-EAC and DGTD-EACe produce similar solutions. For this set of simulations, $E(t)=$ $2 e^{-(t-1.5)^{2} / 0.13} \cos (15[t-1.5])$ and $\Phi_{x}=\Phi_{z}=0$ (normal incidence). The duration of the simulations is 7.5. The computation domains for the DGTD-EAC and DGTD-EACe are $[0 ; 0.5] \times[0 ; 2] \times[0 ; 0.5]$ and $[0 ; 0.5] \times[0 ; 1.5] \times[0 ; 0.5]$, $S^{\mathrm{TF} / \mathrm{SF}}$ is located at $y=1.5$ for the DGTD-EAC. The numbers of the elements in the computation domains are 3357 and 2457. The polynomial order changes from $p=2$ to $p=5$. The time step size changes between $1.38 \times 10^{-3}$ (for $p=2$ ) and $3.4 \times 10^{-4}$ (for $p=5$ ). Fig. 9(a) plots $\operatorname{err}(7.5)$ computed on $\Omega_{\mathrm{err}}=[0 ; 0.5] \times[0 ; 1.5] \times[0 ; 0.5]$ against $p$. As expected both errors decrease as $p$ is increased. Fig. 9(b) plots $E_{z}(0.25,1.5,0.255, t)$ computed by the DGTD-EAC and DGTD-EACe and compares those to $E_{z}^{\text {ref }}(0.25,1.5,0.255, t)$ computed by the DGTD-ref $(p=2)$. The result obtained by the DGTD-EAC is closer to the reference one because both the DGTD-EAC and DGTD-ref use the TF/SF excitation with the same $S^{\mathrm{TF} / \mathrm{SF}}$.

Next, it is shown that $\mathbf{L}_{\mathrm{A}}$ can be located very close to the profile without loss of accuracy. For this set of simulations, $E(t)=$ $2 e^{-(t-1.5)^{2} / 0.13} \cos (15[t-1.5])$ and $\Phi_{x}=\Phi_{z}=0$. The duration of the simulations is 7.5. The computation domains for the DGTD-EAC, DGTD-EACe, DGTD-ABC, and DGTDPML are reduced to $[0 ; 0.5] \times[0 ; 0.7] \times[0 ; 0.5], \quad[0 ; 0.5] \times$ $[0 ; 0.6] \times[0 ; 0.5], \quad[0 ; 0.5] \times[0 ; 0.7] \times[0 ; 0.5]$, and $[0 ; 0.5] \times$ $[0 ; 0.7+d] \times[0 ; 0.5] . S^{\mathrm{TF} / \mathrm{SF}}$ for the DGTD-EAC, DGTD$\mathrm{ABC}$, and DGTD-PML is located at $y=0.6$. The numbers of the elements in the computation domains are 1097, 853, 1097, $1603(d=0.25)$, and $2091(d=0.5)$. The polynomial order is set to $p=3$ and the time step size is $8.4 \times 10^{-4}$. Fig. 10(a) plots $E_{z}(0.35,0.6,0.26, t)$ and $E_{z}^{\text {ref }}(0.35,0.6,0.26, t)$. The figure clearly shows that results obtained by the DGTD-ABC and DGTD-PML with thin PML $(d=0.25)$ differ significantly from 


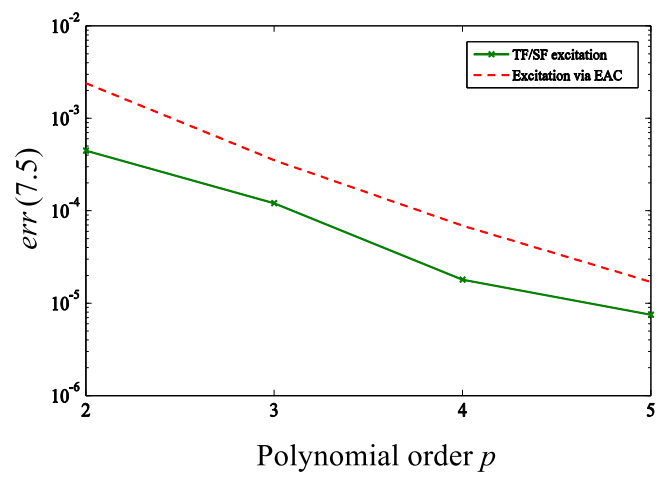

(a)

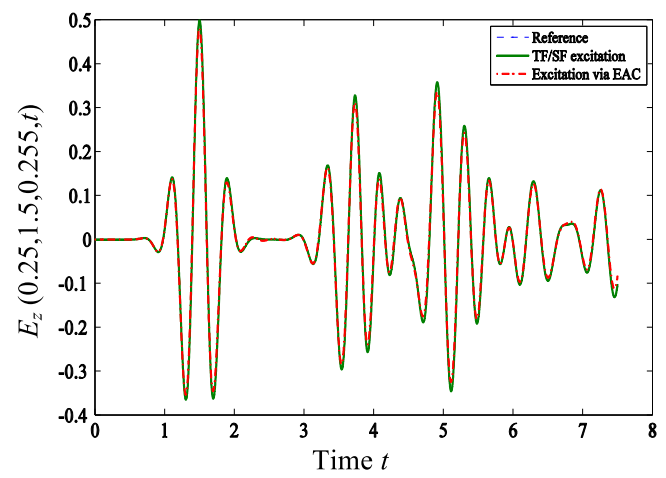

(b)

Fig. 9. (a) err(7.5) computed by DGTD-EAC and DGTD-EACe versus $p$. (b) $E_{z}(0.25,1.5,0.255, t)$ computed by DGTD-EAC, DGTD-EACe, and DGTD-ref.

the reference result. Especially the difference becomes more clear after the excitation leaves the computation domain, between $t=3.5$ and $t=6$. Fig. 10(b) provides a detailed view of the plot in Fig. 10(a) around $t=7$. The solution obtained with the thick PML $(d=0.5)$ is rather accurate, but it is not better than the one obtained by the DGTD-EAC or DGTD-EACe. The $\mathrm{CPU}$ times and memory requirements are $603 \mathrm{~s}$ and $276 \mathrm{MB}$ for the DGTD-EAC, $540 \mathrm{~s}$ and $261 \mathrm{MB}$ for the DGTD-EACe, $501 \mathrm{~s}$ and $111 \mathrm{MB}$ for the DGTD-ABC, $813 \mathrm{~s}$ and $139 \mathrm{MB}$ for the DGTD-PML with $d=0.25$, and $1028 \mathrm{~s}$ and $172 \mathrm{MB}$ for the DGTD-PML with $d=0.5$. Despite the larger memory requirement of the DGTD-EAC and DGTD-EACe, the CPU times correlate with the number of the elements in the computation domains. This demonstrates that the EAC truncations, which require smaller computation domains, are more efficient than the PML and also that the TF/SF excitation implemented on the EAC is more efficient than the TF/SF excitation implemented on $S^{\mathrm{TF} / \mathrm{SF}}$ as expected.

Next, the dependence of error on the simulation duration is characterized. For this set of simulations

$$
E(t)= \begin{cases}0, & 0 \leq t<0.2 \\ s(t)\left(3[m(t)]^{2}-2[m(t)]^{3}\right), & 0.2 \leq t<2 \\ s(t), & 2 \leq t<13 \\ s(t)\left(3[n(t)]^{2}+2[n(t)]^{3}\right), & 13 \leq t<15 \\ 0, & t \geq 15\end{cases}
$$

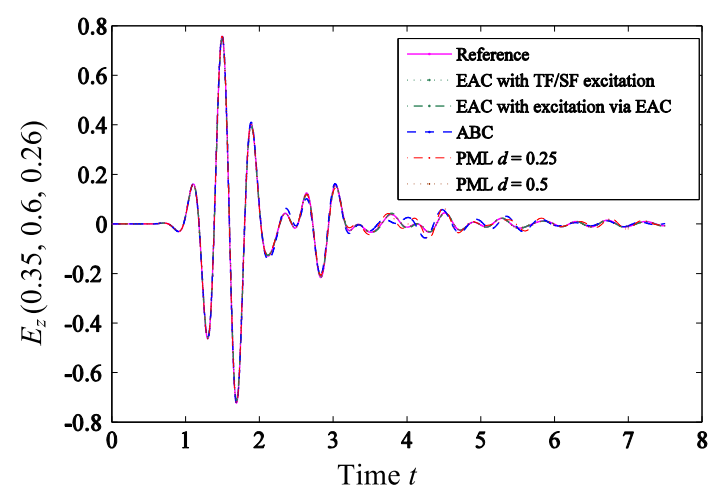

(a)

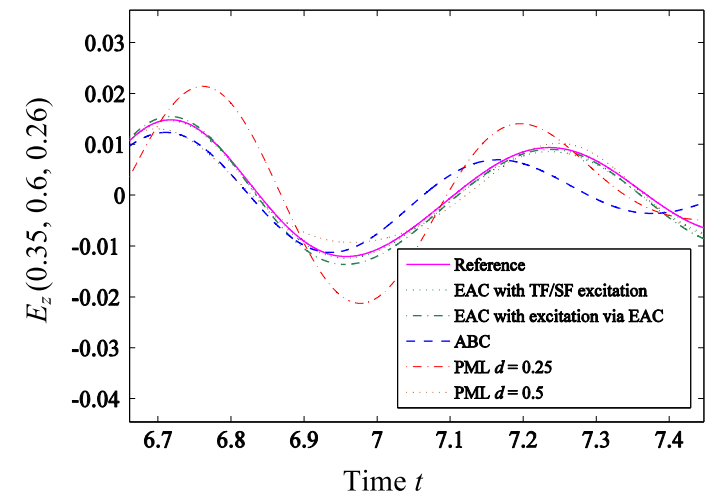

(b)

Fig. 10. $E_{z}(0.35,0.6,0.26, t)$ computed by DGTD-EAC, DGTD-EACe, DGTD-ABC, DGTD-PML, and DGTD-ref. (a) For $t$ between 0 and 8 . (b) For around $t=7$.

where $s(t)=2 \cos (10[t-0.2]), m(t)=(t-0.2) / 1.8$, and $n(t)=(t-15) / 2$, and $\Phi_{x}=\Phi_{z}=0$. The duration of the simulations is 25 . The computation domains for the DGTD-EAC and DGTD-PML with $d=0.5$ are $[0 ; 0.5] \times[0 ; 0.7] \times[0 ; 0.5]$, and $[0 ; 0.5] \times[0 ; 0.7+d] \times[0 ; 0.5] . S^{\mathrm{TF} / \mathrm{SF}}$ is located at $y=$ 0.6 . The numbers of the elements in the computation domains are 1097 and 2091. The polynomial order is set to $p=3$ and the time step size is $8.4 \times 10^{-4}$, which results in a total of 35819 time steps. The error $\operatorname{err}(t)$ is computed on $\boldsymbol{\Omega}_{\mathrm{err}}=[0 ; 0.5] \times[0 ; 0.7] \times[0 ; 0.5]$. Fig. 11 plots $\operatorname{err}(t)$; it is clearly seen that in contrast with the EAC truncation, the error of the PML truncation constantly grows throughout the whole simulation. Even the absence of excitation $(t>15)$ has no effect on this tendency. This means that the accuracy of long duration simulations could be affected by the PML-induced error. Thus, the EAC is a more reliable domain truncation technique, especially for long duration simulations. The CPU times and memory requirements are $2205 \mathrm{~s}$ and $401 \mathrm{MB}$ for the DGTD-EAC, and $4420 \mathrm{~s}$ and $211 \mathrm{MB}$ for the DGTD-PML. Again, the difference in CPU times correlates with the size of the computation domains.

Next, the accuracy of the DGTD-EACe is verified against the commercial solver COMSOL, which implements the frequency-domain FEM. For this simulation, $E(t)=$ $2 e^{-(t-1.5)^{2} / 0.13} \cos (15[t-1.5])$, which covers the frequency 


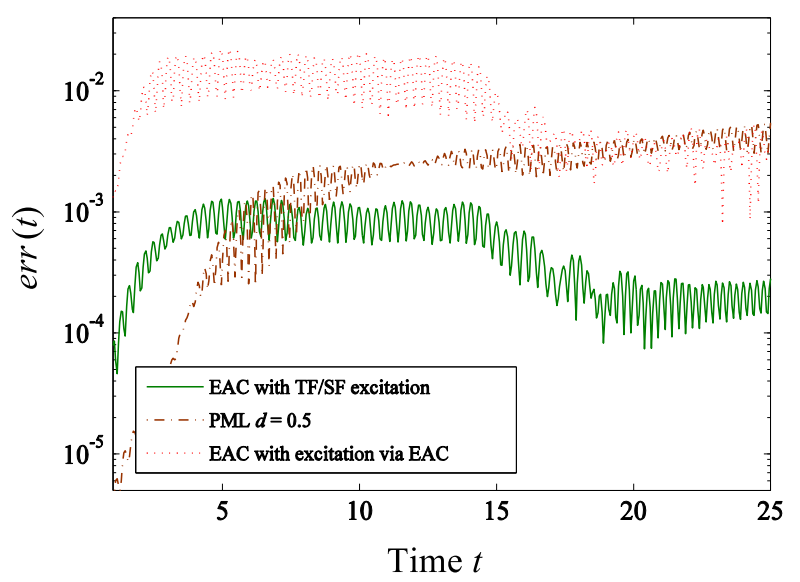

Fig. 11. err $(t)$ computed by DGTD-EAC, DGTD-EACe, and DGTD-PML versus $t$.

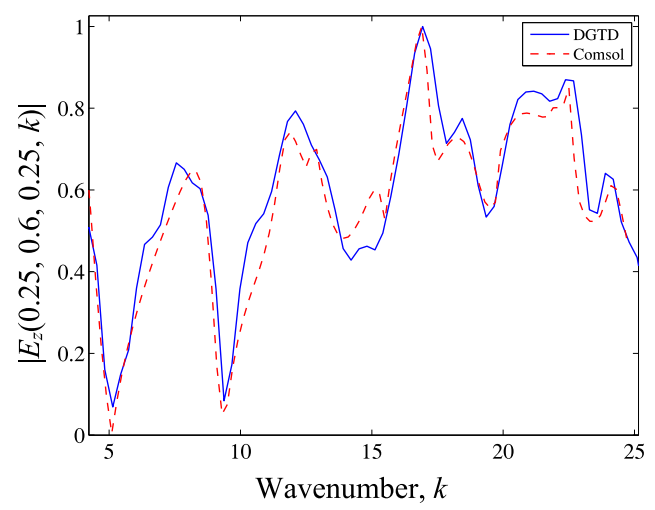

Fig. 12. Fourier transform of $E_{z}(0.25,0.6,0.25, t)$ computed by the DGTD-EACe [after normalization by the Fourier transform of $E(t)$ ] and $\tilde{E}_{z}(0.25,0.6,0.25, k)$ computed by COMSOL.

band $k \in[4,25]$, and $\Phi_{x}=0.25$ and $\Phi_{z}=0$. As explained in Section II-B, each frequency component of the excitation has its own direction of arrival when the constant wavenumber approach is used to treat an oblique incidence. This means that $\theta^{\text {inc }}=0$, and $\varphi^{\text {inc }}$ varies between $7.2^{\circ}$ for $k=25$ and $51.7^{\circ}$ for $k=4$ (see Fig. 1). The duration of the simulation is 7.5. The computation domain is $[0 ; 0.5] \times[0 ; 0.6] \times[0 ; 0.5]$. The number of elements in the computation domain is 853 , the polynomial order is set to $p=3$, and the time step size is $8.4 \times 10^{-4}$. The time-domain electric field $E_{z}(0.25,0.6,0.25, t)$ is computed by the DGTD-EACe. For the COMSOL simulations, the domain truncation and excitation are implemented using the "port" boundary condition. A total of 106 simulations are executed to cover the frequency band $k \in[4,25]$ with 0.2 step. The relation between $\left\{\theta^{\text {inc }}, \varphi^{\text {inc }}\right\}$ (direction of arrival) and frequency of excitation is applied for each value of $k$. The incident electric field has the unit amplitude. The frequency-domain electric field $\tilde{E}_{z}(0.25,0.6,0.25, k)$ is computed by COMSOL. Fig. 12 compares the Fourier transform of $E_{z}(0.25,0.6,0.25, t)$ (computed by the DGTD-EACe), which is normalized by the Fourier transform of $E(t)$, to $\tilde{E}_{z}(0.25,0.6,0.25, k)$ (computed by COMSOL). Good agreement is observed between the results, demonstrating the reliability of the DGTD with the EACs and

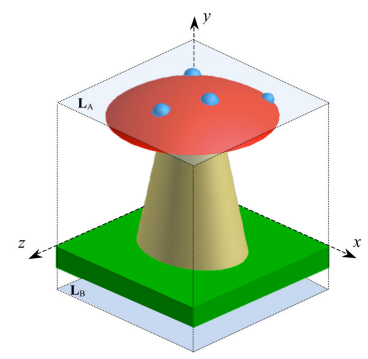

(a)

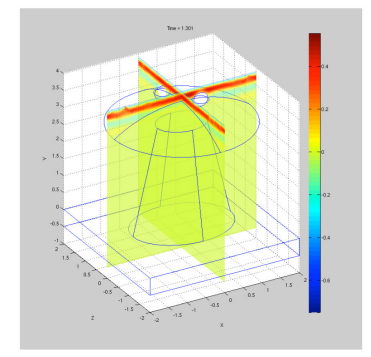

(c)

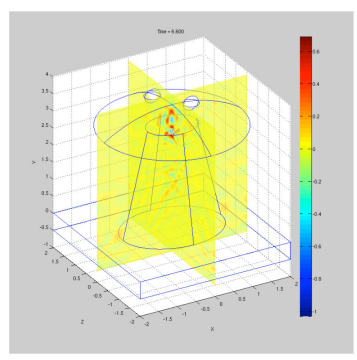

(e)

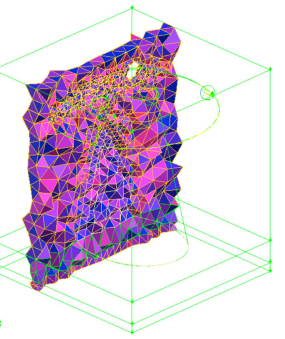

(b)

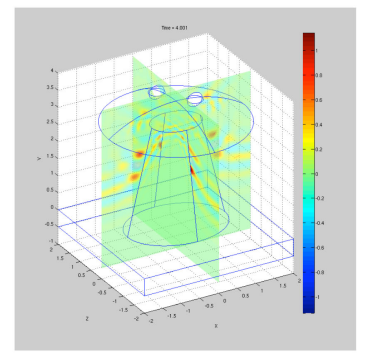

(d)

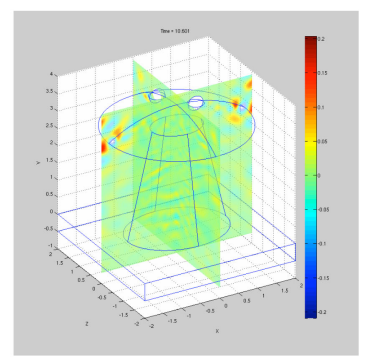

(f)
Fig. 13. (a) Computation domain for the mushroom-shaped grating. (b) Discretization of the computation domain. Snapshots of $E_{z}(\mathbf{r}, t)$ at (c) $t=1.3$, (d) $t=4$, (d) $t=6.6$, and (f) $t=10.6$.

(oblique) PCBs. The CPU time and memory requirements are $930 \mathrm{~s}$ and $309 \mathrm{MB}$ for the DGTD-EACe, and $7200 \mathrm{~s}$ and $10.6 \mathrm{~GB}$ for COMSOL. Note that the CPU time for COMSOL is the total time required to run all 106 frequency-domain simulations.

\section{B. Large and Complex Geometry}

In this section, applicability of the proposed DGTD with the EACs to the characterization of electromagnetic field interactions on complex geometries is demonstrated. The computation domain of the grating is shown in Fig. 13(a). The mushroomshaped structure is centered on a plane substrate. The substrate is made of dielectric with $\varepsilon=2.5$ and has a thickness of 0.5 . The mushroom stem is made of dielectric with $\varepsilon=7.5$. The stem's bottom base is an ellipse with major and minor semiaxes of length 1.3 (in $x$ direction) and 1 (in $z$ direction). The stem's top base is an ellipse with major and minor semiaxes of length 0.65 (in $x$ direction) and 0.5 (in $z$ direction). The stem's height is 3 . The mushroom cap is made of dielectric with $\varepsilon=4.5$. The cap's bottom is a circle with radius 1.8. The cap's height is 0.6 . The cap's top coincides with a sphere centered at $(0,0.6,0)$ with radius 3 . The cap is doped with four spherical PEC inclusions. The radius of each PEC inclusions is 0.2 , and they 
are centered at $(0,3.4,0.9),(0.6,3.5,0),(-0.9,3.2,-0.85)$, and $(0.66,3.1,-1.5)$. The periods in $x$ and $z$ directions are $l_{x}=l_{z}=2$.

The grating is excited by a normally incident plane wave $\left(\Phi_{x}=\Phi_{z}=0\right), E(t)=2 e^{-4(t-1.5)^{2}} \cos (13[t-1.5])$. The duration of the simulation is 13 , the time step size is $8.4 \times 10^{-4}$ resulting in 15432 time steps. The computation domain is $[0 ; 2] \times[-0.7 ; 3.9] \times[0 ; 2] ; \mathbf{L}_{\mathrm{A}}$ with $\operatorname{EAC}(13)$ and $\mathbf{L}_{\mathrm{B}}$ with EAC (12) are located at $y=3.9$ and $y=-0.7$ [see Fig. 13(a)]. The distance between the bottom of the substrate and $\mathbf{L}_{\mathrm{B}}$ is 0.2. The summations in EACs are truncated to 15 terms each, i.e., $N_{n}=N_{m}=7$. The mesh has $N_{e}=80150$ elements in the computation domain. The polynomial order is $p=2$ resulting in 4809000 unknowns.

Fig. 13(c)-(f) show snapshots of $E_{z}(\mathbf{r}, t)$ inside the computation domain at different times. Although the simulation of the mushroom-shaped grating is designed for demonstration purposes. Fig. 13 allows to observe some interesting effects. It is seen that the stem acts as a guiding structure for downward travelling waves [see Fig. 13(d)]. A resonance is formed inside the cap [see Fig. 13(e)]. A rise of the field amplitude could be observed outside the cap [see Fig. 13(f)].

\section{CONCLUSION}

Time-domain periodic and EACs under obliquely incident fields are derived and their discretization within a DGTD method is described. The resulting solver allows for accurate and efficient analysis of transient electromagnetic wave interactions on 3-D periodic structures. Indeed, numerical results demonstrate that the domain truncation with EACs is more accurate and efficient than with approximate absorbing boundary conditions or perfectly matched layers.

\section{ACKNOWLEDGMENT}

The authors would like to thank KAUST Supercomputing Laboratory (KSL) for providing computational resources needed to carry out the numerical experiments.

\section{REFERENCES}

[1] M. Neviere and E. Popov, Light Propagation in Periodic Media: Differential Theory and Design. New York, NY, USA: Marcel Dekker, 2003.

[2] Y. K. Sirenko and S. Strom, Eds., Modern Theory of Gratings. Resonant Scattering: Analysis Techniques and Phenomena. New York, NY, USA: Springer-Verlag, 2010.

[3] A. Lehmuskero, I. Vartiainen, T. Saastamoinen, T. Alasaarela, and M. Kuittinen, "Absorbing polarization selective resonant gratings," Opt. Express, vol. 18, no. 26, pp. 27 270-27 279, Dec. 2010.

[4] Z. Lu, S. Shi, C. A. Schuetz, J. A. Murakowski, and D. W. Prather, "Threedimensional photonic crystal flat lens by full 3D negative refraction," Opt. Express, vol. 13, no. 15, pp. 5592-5599, Jul. 2005.

[5] M. Amin, M. Farhat, and H. Bagci, "A dynamically reconfigurable Fano metamaterial through graphene tuning for switching and sensing applications," Sci. Rep., vol. 3, 2013, Art. no. 2105.

[6] M. Amin, M. Farhat, and H. Bagci, "A nonlinear plasmonic resonator for three-state all-optical switching," Opt. Express, vol. 22, no. 6, pp. 69666975, Mar. 2014.

[7] A. Taflove and S. C. Hagness, Computational Electrodynamics. Norwood, MA, USA: Artech House, 2005.

[8] J.-M. Jin, The Finite Element Method in Electromagnetics. New York, NY, USA: Wiley, 2014.
[9] D. Jiao, A. A. Ergin, B. Shanker, E. Michielssen, and J.-M. Jin, "A fast higher-order time-domain finite element-boundary integral method for 3-D electromagnetic scattering analysis," IEEE Trans. Antennas Propag., vol. 50, no. 9, pp. 1192-1202, Sep. 2002.

[10] D. Jiao, J.-M. Jin, E. Michielssen, and D. J. Riley, "Time-domain finiteelement simulation of three-dimensional scattering and radiation problems using perfectly matched layers," IEEE Trans. Antennas Propag., vol. 51, no. 2, pp. 296-305, Feb. 2003.

[11] J. S. Hesthaven and T. Warburton, Nodal Discontinuous Galerkin Methods: Algorithms, Analysis, and Applications. New York, NY, USA: Springer-Verlag, 2008

[12] J. Chen and Q. H. Liu, "Discontinuous Galerkin time-domain methods for multiscale electromagnetic simulations: A review," Proc. IEEE, vol. 101, no. 2, pp. 242-254, Feb. 2013.

[13] J.-H. Lee, J. Chen, and Q. H. Liu, "A 3-D discontinuous spectral element time-domain method for Maxwell's equations," IEEE Trans. Antennas Propag., vol. 57, no. 9, pp. 2666-2674, Sep. 2009.

[14] L. E. Tobon, Q. Ren, and Q. H. Liu, "A new efficient 3D discontinuous Galerkin time domain (DGTD) method for large and multiscale electromagnetic simulations," J. Comput. Phys., vol. 283, pp. 374-387, 2015.

[15] P. Li, L. J. Jiang, and H. Bagci, "Transient analysis of dispersive powerground plate pairs with arbitrarily shaped antipads by the DGTD method with wave port excitation," IEEE Trans. Electromagn. Compat., vol. 59 , no. 1, pp. 172-183, Feb. 2017.

[16] P. Li, L. J. Jiang, and H. Bagci, "Discontinuous Galerkin time-domain analysis of power-ground planes taking into account decoupling capacitors," IEEE Trans. Compon. Packag. Manuf. Technol., vol. 7, no. 9, pp. 14761485, Sep. 2017.

[17] P. Li, Y. Dong, M. Tang, J. Mao, L. J. Jiang, and H. Bagci, "Transient thermal analysis of 3-D integrated circuits packages by the DGTD method," IEEE Trans. Compon. Packag. Manuf. Technol., vol. 7, no. 6, pp. 862-871, Jun. 2017.

[18] P. Li, L. J. Jiang, and H. Bagci, "Transient analysis of lumped circuit networks-loaded thin wires by DGTD method," IEEE Trans. Antennas Propag., vol. 64, no. 6, pp. 2358-2369, Jun. 2016.

[19] P. Li, Y. Shi, L. J. Jiang, and H. Bagci, "DGTD analysis of electromagnetic scattering from penetrable conductive objects with IBC," IEEE Trans. Antennas Propag., vol. 63, no. 12, pp. 5686-5697, Dec. 2015.

[20] K. Sirenko, M. Liu, and H. Bagci, "Incorporation of exact boundary conditions into a discontinuous Galerkin finite element method for accurately solving 2D time-dependent Maxwell equations," IEEE Trans. Antennas Propag., vol. 61, no. 1, pp. 472-477, Jan. 2013.

[21] K. Busch, M. Konig, and J. Niegemann, "Discontinuous Galerkin methods in nanophotonics," Laser Photon. Rev., vol. 5, no. 6, pp. 773-809, 2011.

[22] M. Liu, K. Sirenko, and H. Bagci, "An efficient discontinuous Galerkin finite element method for highly accurate solution of Maxwell equations," IEEE Trans. Antennas Propag., vol. 60, no. 8, pp. 3992-3998, Aug. 2012

[23] T. Lu, P. Zhang, and W. Cai, "Discontinuous Galerkin methods for dispersive and lossy Maxwell's equations and PML boundary conditions," $J$. Comput. Phys., vol. 200, no. 2, pp. 549-580, 2004.

[24] P. Li, Y. Shi, L. J. Jiang, and H. Bagci, "A hybrid time-domain discontinuous Galerkin-boundary integral method for electromagnetic scattering analysis," IEEE Trans. Antennas Propag., vol. 62, no. 5, pp. 2841-2846, May 2014.

[25] A. Glaser and V. Rokhlin, "A new class of highly accurate solvers for ordinary differential equations," J. Sci. Comput., vol. 38, no. 3, pp. 368 399, Mar. 2009.

[26] D. Givoli, "Non-reflecting boundary conditions," J. Comput. Phys. vol. 94, no. 1, pp. 1-29, 1991.

[27] S. V. Tsynkov, "Numerical solution of problems on unbounded domains. A review," Appl. Numer. Math., vol. 27, no. 4, pp. 465-532, 1998.

[28] D. Givoli, "High-order local non-reflecting boundary conditions: A review," Wave Motion, vol. 39, no. 4, pp. 319-326, 2004.

[29] Y. Sirenko, S. Strom, and N. P. Yashina, Modeling and Analysis of Transient Processes in Open Resonant Structures. New Methods and Techniques. New York, NY, USA: Springer-Verlag, 2007.

[30] K. Y. Sirenko, Y. K. Sirenko, and N. P. Yashina, "Modeling and analysis of transients in periodic gratings. I. Fully absorbing boundaries for 2-D open problems," J. Opt. Soc. Amer. A, vol. 27, no. 3, pp. 532-543, Mar. 2010.

[31] K. Sirenko, V. Pazynin, Y. K. Sirenko, and H. Bagci, “An FFT-accelerated FDTD scheme with exact absorbing conditions for characterizing axially symmetric resonant structures," Prog. Electromagn. Res., vol. 111, pp. 331-364, 2011. 
[32] A. E. Yilmaz, D. S. Weile, B. Shanker, J.-M. Jin, and E. Michielssen, "Fast analysis of transient scattering in lossy media," IEEE Antennas Wireless Propag. Lett., vol. 1, pp. 14-17, 2002.

[33] H. Bagci, A. E. Yilmaz, and E. Michielssen, "FFT-accelerated MOTbased solution of time-domain BLT equations," in Proc. IEEE Int. Symp. Antennas Propag., Jul. 2006, pp. 1175-1184.

[34] H. Bagci, A. E. Yilmaz, and E. Michielssen, "An FFT-accelerated timedomain multiconductor transmission line simulator," IEEE Trans. Electromagn. Compat., vol. 52, no. 1, pp. 199-214, Feb. 2010.

[35] K. Y. Sirenko, Y. K. Sirenko, and N. P. Yashina, "Modeling and analysis of transients in periodic gratings. II. Resonant wave scattering," J. Opt. Soc. Amer. A, vol. 27, no. 3, pp. 544-552, Mar. 2010

[36] K. Sirenko, V. Pazynin, Y. Sirenko, and H. Bagci, "Compression and radiation of high-power short RF pulses. I. Energy accumulation in directflow waveguide compressors," Prog. Electromagn. Res., vol. 116, pp. 239270,2011

[37] K. Sirenko, V. Pazynin, Y. Sirenko, and H. Bagci, "Compression an radiation of high- power short RF pulses. II. A novel antenna array design with combined compressor/radiator elements," Prog. Electromagn. Res., vol. 116, pp. 271-296, 2011.

[38] F. Yang, J. Chen, R. Qiang, and A. Elsherbeni, "A simple and efficien FDTD/PBC algorithm for scattering analysis of periodic structures," Radio Sci., vol. 42, no. 4, 2007, Art. no. RS4004.

[39] A. Perov, Y. Sirenko, and N. Yashina, "Explicit conditions for virtual boundaries in initial boundary value problems in the theory of wave scattering," J. Electromagn. Waves Appl., vol. 13, no. 10, pp. 1343-1371, 1999.

[40] G. Granet, P. Melezhik, K. Sirenko, and N. Yashina, "Time-and-frequency domains approach to data processing in multiwavelength optical scatterometry of dielectric gratings," J. Opt. Soc. Amer. A, vol. 30, no. 3, pp. 427-436, Mar. 2013.
[41] G. Tang, R. L. Panetta, and P. Yang, "Application of a discontinuous Galerkin time domain method to simulation of optical properties of dielectric particles," Appl. Opt., vol. 49, no. 15, pp. 2827-2840, May 2010.

[42] X. Ji, T. Lu, W. Cai, and P. Zhang, "Discontinuous Galerkin time domain (DGTD) methods for the study of 2-D waveguide-coupled microring resonators," J. Lightw. Technol., vol. 23, no. 11, pp. 3864-3874, Nov. 2005.

[43] K. Stannigel, M. Konig, J. Niegemann, and K. Busch, "Discontinuous Galerkin time-domain computations of metallic nanostructures," Opt. Express, vol. 17, no. 17, pp. 14 934-14 947, Aug. 2009.

[44] L. E. R. Petersson and J.-M. Jin, "A two-dimensional time-domain finite element formulation for periodic structures," IEEE Trans. Antennas Propag., vol. 53, no. 4, pp. 1480-1488, Apr. 2005.

[45] J. A. Roden, S. D. Gedney, M. P. Kesler, J. G. Maloney, and P. H. Harms, "Time-domain analysis of periodic structures at oblique incidence: Orthogonal and nonorthogonal FDTD implementations," IEEE Trans. Microw. Theory Techn., vol. 46, no. 4, pp. 420-427, Apr. 1998

[46] "Encyclopedia of cubature formulas," 2005. [Online]. Available: http:// nines.cs.kuleuven.be/research/ecf/ecf.html. Accessed on: 2016.

[47] B. T. Helenbrook, "On the existence of explicit $h p$-finite element methods using Gauss-Lobatto integration on the triangle," SIAM J. Numer. Anal., vol. 47 , no. 2 , pp. 1304-1318, 2009.

Authors' photographs and biographies not available at the time of publication. 